\title{
Motion and structure of atmospheric mesoscale baroclinic vortices: dry air and weak environmental shear
}

\author{
Eileen Päschke ${ }^{1}$, Patrik Marschalik ${ }^{2}$, Antony Z. Owinoh $\ddagger$ and Rupert Klein ${ }^{2} \dagger$ \\ ${ }^{1}$ Institut für Mathematik, Technische Universität Berlin, 14195 Berlin, Germany \\ ${ }^{2}$ Fachbereich Mathematik \& Informatik, Freie Universität Berlin, 10632 Berlin, Germany
}

(Received 12 November 2010; revised 6 March 2012; accepted 12 March 2012; first published online 14 May 2012)

\begin{abstract}
A strongly tilted, nearly axisymmetric vortex in dry air with asymmetric diabatic heating is analysed here by matched asymptotic expansions. The vortex is in gradient wind balance, with vortex Rossby numbers of order unity, and embedded in a quasigeostrophic (QG) background wind with weak vertical shear. With wind speeds of $60-120 \mathrm{~km} \mathrm{~h}^{-1}$, such vortices correspond to tropical storms or nascent hurricanes according to the Saffir-Simpson scale. For asymmetric heating, nonlinear coupling of the evolution equations for the vortex tilt, its core structure, and its influence on the QG background is found. The theory compares well with the established linear theory of precessing quasi-modes of atmospheric vortices, and it corroborates the relationship between vortex tilt and asymmetric potential temperature and vertical velocity patterns as found by Jones $(Q . J$. R. Meteorol. Soc., vol. 121, 1995, pp. 821-851) and Frank \& Ritchie (Mon. Weath. Rev., vol. 127, 1999, pp. 2044-2061) in simulations of adiabatic tropical cyclones. A relation between the present theory and the local induction approximation for three-dimensional slender vortex filaments is established.
\end{abstract}

Key words: atmospheric flows, meteorology, quasi-geostrophic flows

\section{Introduction}

\subsection{Related earlier work}

There is a large body of literature, starting with Stewart (1943) and Morikawa (1960) (see also Egger 1992; Reznik 1992; Reznik \& Kizner 2007a,b, and references therein) that investigates the dynamics of concentrated atmospheric vortices in the limit of pointwise vorticity singularities in two-dimensional or multilayer shallow-flow models. The related theories focus on the vortex motion but do not investigate the vortex core structure.

On the other hand, the core structure of such vortices is often modelled using Eliassen's balanced vortex model and extensions thereof (see e.g. Eliassen 1952; Charney \& Eliassen 1964; Schubert \& Hack 1983; Emanuel 1991; Wirth \& Dunkerton 2009, and references therein), which describes the evolution of an axisymmetric,

$\dagger$ Email address for correspondence: rupert.klein@math.fu-berlin.de

$\$$ Dr Owinoh passed away while this paper was under revision. 
upright vortex in gradient wind balance, i.e. in a regime where the radial pressure gradient is balanced to leading order by the centripetal and Coriolis accelerations. The theories developed provide no information regarding the vortex motion.

Asymptotic theories that resolve the vortex core structure and describe its coupling to the environmental flow and/or the motion of its centre were developed by Ling \& Ting (1988) in the limit of geostrophic balance, by Smith \& Ulrich (1990) and Smith (1991) for two-dimensional flow on a $\beta$-plane, and by Reznik \& Grimshaw (2001) and McWilliams, Graves \& Montgomery (2003) in the context of shallow-water theory. By restricting to two-dimensional shallow-fluid models, these theories cannot address the vertical structure of the flow and, especially, a possible tilt of the vortex axis.

Shapiro \& Montgomery (1993) develop a linearized asymptotic theory that is close, in terms of its principal aim, to the present work. It describes the evolution of threedimensional balanced perturbations on an upright, axisymmetric vortex in gradient wind balance. It includes circumferential Fourier-mode-one perturbations that can be interpreted as a weak vortex tilt with horizontal displacements small compared with the vortex core radius. The authors also suggest extensions of their linearized perturbation analysis to include weakly nonlinear effects.

The dynamics of vortex tilt, and especially the resilience of a vortex to background vertical shear, has been analysed by Reasor \& Montgomery (2001) and Reasor, Montgomery \& Grasso (2004) through both theoretical analyses and numerical simulations based on the primitive flow equations. Their linear theory of vortex Rossby waves (VRWs) (see Montgomery \& Kallenbach 1997) reveals a damping mechanism for the vortex tilt dynamics and allows them to explain the stabilization of an atmospheric vortex against vertical shear of the environmental flow. At the same time, these developments reveal weakly damped linear 'quasi-modes' which, in a suitable regime of vortex Rossby numbers, can be interpreted as small-amplitude precessions of the entire vortex about its unperturbed vertical axis. Surprisingly, the theory - although based on linearizations - yields very good comparison with fully nonlinear simulations when relative deviations from the unperturbed state are of order unity, i.e. when the horizontal displacements of the vortex core are comparable to its characteristic diameter. In a first application the present analysis corroborates the existence of such large displacement precessions within the gradient wind regime and yields agreement of the predicted precession frequencies for vortex Rossby numbers of order unity. See also related numerical simulations by Jones $(1995,2004)$.

\subsection{Summary of the present approach and main results}

In this paper we consider concentrated nearly axisymmetric strongly tilted baroclinic vortices in gradient wind balance that are embedded in a quasi-geostrophic (QG) farfield flow with weak vertical shear. We restrict to the case of dry air with an ideal-gas equation of state but include the principal effects of asymmetric diabatic sources in the vortex core.

Let $\boldsymbol{X}_{0}(t)$ denote the overall horizontal position of the vortex measured in terms of a synoptic length scale, $L_{s y n}$, and let $\boldsymbol{X}^{(1)}(t, z)=\boldsymbol{i} X^{(1)}(t, z)+\boldsymbol{j} Y^{(1)}(t, z)$ describe the horizontal displacement of the vortex centre from that large-scale position at height $z$, measured in units of a typical vortex core diameter, $L_{m e s}$. Then $\delta=L_{m e s} / L_{s y n} \ll 1$ for concentrated vortices, and the horizontal position of the vortex centre at height $z$ measured in units of $L_{s y n}$ is (cf. figure 1)

$$
\boldsymbol{X}(t, z) \equiv X(t, z) \boldsymbol{i}+Y(t, z) \boldsymbol{j}=\boldsymbol{X}_{0}(t)+\delta \boldsymbol{X}^{(1)}(t, z) .
$$




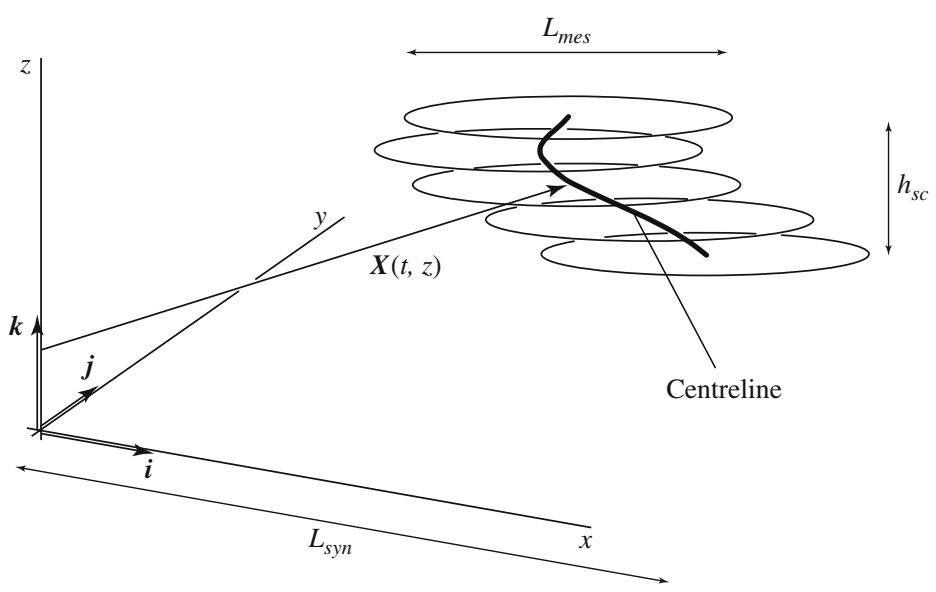

FIGURE 1. Tilted concentrated mesoscale vortex of characteristic height $h_{s c}$ and typical core size $L_{m e s}$ embedded in a synoptic-scale $\left(L_{s y n}\right)$ environment, where $h_{s c} \ll L_{m e s} \ll L_{s y n}$.

Thus we allow for horizontal displacements comparable to a typical vortex core size of $L_{\text {mes }} \sim 150 \mathrm{~km}$. This amounts to very strong tilt since the characteristic height of the vortex is comparable to $h_{s c} \sim 10 \mathrm{~km}$. With $\boldsymbol{X}_{0}$ independent of $z$ we avoid unrealistically large tilt with horizontal displacements much larger than the vortex core size.

Let $u_{\theta}$ denote the primary, possibly baroclinic, circulation of the vortex with total strength $\Gamma(t, z)$. The gradient wind regime involves the dominant three-term balance of the pressure gradient, centrifugal acceleration and Coriolis terms and implies vortex Rossby numbers of order unity: with $p, \rho$, and $f_{0}$ denoting pressure, density, and the Coriolis parameter, respectively, we have $\partial p / \partial r \sim \rho u_{\theta}^{2} / r \sim \rho f_{0} u_{\theta}$ in the gradient wind regime, with $r$ the horizontal distance from the centreline. Then, with $L_{m e s}$ of the order of a characteristic vortex radius, the comparison of the latter two terms yields an estimate for the vortex Rossby number, $R o_{v}=u_{\theta} / f_{0} L_{m e s}=O(1)$. Accordingly we let $R o_{v}=O(1)$ as $\delta \rightarrow 0$ in the subsequent asymptotic analysis.

Motivated by Ting and co-workers' approach to the dynamics of slender fluid vortices (see Ling \& Ting 1988; Ting, Klein \& Knio 2007) we derive nonlinear coupled evolution equations for the vortex core structure, the vortex tilt, and the QG outer flow through matched asymptotic expansions of the compressible flow equations in three space dimensions.

Specifically, we consider in $\S 3$ the QG far-field flow with an embedded tilted singular baroclinic line vortex. Given the vortex centreline from (1.1), the vortex circulation, $\Gamma(t, z)$, and the atmospheric background state characterized by the density, $\rho_{0}(z)$, and the mean perturbation potential temperature stratification, $\Theta_{1}(z)$, we have the perturbed quasi-geostrophic far-field flow

$$
\boldsymbol{u}=-\nabla_{\|}^{\perp} \psi,
$$

where $\nabla_{\|}$is the horizontal gradient, and

$$
\psi=\psi_{0}^{2 d}+\psi_{1}^{2 d}+\psi_{2}^{*}
$$


is the QG streamfunction with the local vortex-induced components

$$
\psi_{0}^{2 d}=-\frac{\Gamma}{2 \pi} \ln r \quad \text { and } \quad \psi_{1}^{2 d}=M_{0} r^{2}(\ln r-1)+\delta M_{1} r \ln r-\delta^{2} M_{2} .
$$

Here $r(t, \boldsymbol{x}, z)=|\boldsymbol{x}-\boldsymbol{X}(t, z)|$ is the horizontal distance from the centreline and the coefficients $M_{i}$ depend on $\rho_{0}(z), \Theta_{1}(z)$, and $\Gamma(t, z)$ through (3.22) given below. The regularized streamfunction, $\psi_{2}^{*}$, satisfies

$$
\left(\nabla_{\|}^{2}+\mathscr{L}_{z}\right) \psi_{2}^{*}=-\mathscr{L}_{z} \psi_{1}^{2 d}-q_{r} \quad \text { with } \mathscr{L}_{z}[\cdot]=\frac{f_{0}^{2}}{\rho_{0}} \frac{\partial}{\partial z}\left(\frac{\rho_{0}}{\mathrm{~d} \Theta_{1} / \mathrm{d} z} \frac{\partial \cdot}{\partial z}\right) .
$$

The regular part of the potential vorticity, $q_{r}$, which includes planetary rotation, is advected with the horizontal flow, i.e.

$$
\left(\frac{\partial}{\partial t}+\boldsymbol{u} \cdot \nabla_{\|}\right) q_{r}=0 .
$$

In $\S 4$ we show that changes in the leading-order axisymmetric circumferential flow velocity, $u_{\theta}(t, \widehat{r}, z)$, in the vortex core are described by the core structure evolution equation

$$
\frac{\partial u_{\theta}}{\partial t}+w_{0} \frac{\partial u_{\theta}}{\partial z}+u_{r, 00}\left(\frac{\partial u_{\theta}}{\partial \widehat{r}}+\frac{u_{\theta}}{\widehat{r}}+f_{0}\right)=-u_{r, *}\left(\frac{u_{\theta}}{\widehat{r}}+f_{0}\right),
$$

where

$$
\begin{gathered}
w_{0}=\frac{Q_{\Theta, 0}}{\mathrm{~d} \Theta_{1} / \mathrm{d} z}, \\
u_{r, 00}=-\frac{1}{\widehat{r}} \int_{0}^{\widehat{r}} \frac{r^{\prime}}{\rho_{0}} \frac{\partial}{\partial z}\left(\rho_{0} \frac{Q_{\Theta, 0}}{\mathrm{~d} \Theta_{1} / \mathrm{d} z}\right) \mathrm{d} r^{\prime}, \\
u_{r, *}=\frac{1}{2}\left[\frac{\partial X^{(1)}}{\partial z} \frac{Q_{\Theta, 12}}{\mathrm{~d} \Theta_{1} / \mathrm{d} z}+\frac{\partial Y^{(1)}}{\partial z} \frac{Q_{\Theta, 11}}{\mathrm{~d} \Theta_{1} / \mathrm{d} z}\right] .
\end{gathered}
$$

Here $Q_{\Theta, 0}$ and $Q_{\Theta, 1 k}$ for $k=1,2$ are the axisymmetric and first circumferential Fouriermode amplitudes of the diabatic source term, $u_{r, 00}$ and $w_{0}$ are the axisymmetric parts of the radial and vertical velocities that are directly induced by axisymmetric diabatic heating, $\widehat{r}=r / \delta$ is the stretched radial coordinate resolving the vortex core, and $f_{0}$ is the Coriolis parameter.

The terms on the left of $(1.3 a)$ are analogous to those found in the circumferential momentum balance of an axisymmetric system. The forcing on the right is due to nonlinear interactions of the vortex tilt with asymmetric diabatic heating. The forcing strength, represented by $u_{r, *}$, depends on the relative arrangement of the tilt direction and the diabatic heating asymmetries. Suitable arrangement can spin up or spin down the vortex. This is in agreement with results by Shapiro \& Montgomery (1993) on balanced small perturbations of an axisymmetric, vertically aligned vortex and extends them to vortices with leading-order tilt.

In $\S 5$ we derive the vortex centreline equations of motion

$$
\begin{gathered}
\frac{\mathrm{d} \boldsymbol{X}_{0}}{\mathrm{~d} t}=\boldsymbol{u}_{0}, \\
\frac{\partial \boldsymbol{X}^{(1)}}{\partial t}=\boldsymbol{u}_{0}^{(1 / 2)}+\boldsymbol{X}^{(1)} \cdot\left(\nabla_{\|} \boldsymbol{u}_{0}\right)-\ln \frac{1}{\delta}\left(\boldsymbol{k} \times \boldsymbol{M}_{1}\right)+(\boldsymbol{k} \times \boldsymbol{\Psi}),
\end{gathered}
$$

where $\boldsymbol{u}_{0}$ and $\nabla_{\|} \boldsymbol{u}_{0}$ are the barotropic leading-order regular part of the quasigeostrophic background flow evaluated at $\boldsymbol{X}_{0}(t)$ and its horizontal gradient, 
respectively, $\boldsymbol{u}^{(1 / 2)}$ is a possibly baroclinic contribution to the background flow of $O(\delta)$ (the $(1 / 2)$ superscript will be explained shortly), $\boldsymbol{M}_{1}$ is related to $M_{1}$ from $(1.2 c)$, (3.22), and $\boldsymbol{\Psi}$ is a complex expression involving the vortex core structure, $u_{\theta}(t, \widehat{r}, z)$, the asymmetric heating, and the vortex tilt (see (5.12), (5.10), and (4.33) below).

The main conclusions to be drawn from (1.4) are, first, that in the considered regime with relatively weak heating and tilt-induced vertical motions a vortex cannot sustain strong vertical shear of the background flow within which it is embedded. We must require the leading-order background flow, $\boldsymbol{u}_{0}$, to be barotropic, i.e. independent of $z$. Secondly, there is a self-induced motion of a tilted baroclinic vortex which induces horizontal displacements comparable to the vortex core size (terms involving $\boldsymbol{M}_{1}$ and $\boldsymbol{\Psi})$. See $\S 6$ for a comparison of predictions using (1.4) with established linear theories for small perturbations of an initially vertically aligned vortex by Reasor et al. (2004).

Two remarks of caution are in order: the present theory proceeds independently of whether we assume cyclonic or anticyclonic mean vortex rotation. It does assume, however, that the vortex is stable over the characteristic time scale considered and this may exclude anticyclonic vortices with Rossby numbers larger than unity from consideration because of possible centrifugal instabilities (see e.g. Afanasyev \& Peltier 1998).

Also, the reader will have noticed the occurrence of the logarithm of the perturbation parameter in (1.4). This does not come as a surprise considering established theories for slender fluid vortices (see e.g. Ting et al. 2007). Here we follow Ting and co-workers, who consider such logarithmic terms as of order unity relative to powers of the perturbation parameter. This seems appropriate here as $\delta>1 / 10$ for realistic applications of the present theory (see $\S 7.3$ for further discussion).

For conclusions, limitations of the theory, and an outlook see $\S 7$.

\section{Dimensionless governing equations and distinguished limits}

The inviscid rotating compressible flow equations in the beta-plane approximation,

$$
\begin{gathered}
\frac{\partial \boldsymbol{u}}{\partial t}+\boldsymbol{u} \cdot \nabla_{\|} \boldsymbol{u}+w \frac{\partial \boldsymbol{u}}{\partial z}+\frac{1}{\rho} \nabla_{\|} p+f \boldsymbol{k} \times \boldsymbol{u}=0, \\
\frac{\partial w}{\partial t}+\boldsymbol{u} \cdot \nabla_{\|} w+w \frac{\partial w}{\partial z}+\frac{1}{\rho} \frac{\partial p}{\partial z}=-g, \\
\frac{\partial \rho}{\partial t}+\boldsymbol{u} \cdot \nabla_{\|} \rho+w \frac{\partial \rho}{\partial z}+\rho \nabla_{\|} \cdot \boldsymbol{u}+\rho \frac{\partial w}{\partial z}=0, \\
\frac{\partial \Theta}{\partial t}+\boldsymbol{u} \cdot \nabla_{\|} \Theta+w \frac{\partial \Theta}{\partial z}=Q_{\Theta}, \\
\Theta=\frac{p_{r e f}}{\rho R}\left(\frac{p}{p_{r e f}}\right)^{1 / \gamma},
\end{gathered}
$$

are our point of departure. Here $p, \rho, \Theta, \boldsymbol{u}, w$ are pressure, density, potential temperature, and the horizontal and vertical velocities, and $\gamma$ is the specific heat ratio. The Coriolis parameter is $f=f_{0}+\beta y$ where $y$ denotes the local meridional coordinate and $\beta=$ const. The horizontal gradient is $\nabla_{\|}=\boldsymbol{i} \partial / \partial x+\boldsymbol{j} \partial / \partial y$ with $x$ the zonal coordinate, and the three-dimensional gradient is $\nabla=\nabla_{\|}+\boldsymbol{k} \partial / \partial z$. $Q_{\Theta}$ is a generic diabatic source term.

Table 1 lists general characteristics of the near-tropical atmosphere (latitude $\phi=30^{\circ} \mathrm{N}$ ) which we combine in table 2 to obtain reference values for 
Gravitational acceleration

Coriolis parameter $\left(\phi=30^{\circ} \mathrm{N}\right)$

$(\mathrm{d} f / \mathrm{d} y)_{0}\left(\phi=30^{\circ} \mathrm{N}\right)$

Pressure

Temperature

Brunt-Väisälä frequency

Dry gas constant

Isentropic exponent

$$
\begin{aligned}
& g=9.81 \mathrm{~m} \mathrm{~s}^{-2} \\
& f_{0}=7.3 \times 10^{-5} \mathrm{~s}^{-1} \\
& \beta=2.0 \times 10^{-11} \mathrm{~m}^{-1} \mathrm{~s}^{-1} \\
& p_{\text {ref }}=10^{5} \mathrm{~Pa} \\
& T_{\text {ref }}=300 \mathrm{~K} \\
& N=10^{-2} \mathrm{~s}^{-1} \\
& R=287 \mathrm{~m}^{2} \mathrm{~s}^{-2} \mathrm{~K}^{-1} \\
& \gamma=1.4
\end{aligned}
$$

TABLE 1. Characteristic atmospheric flow parameters

$\begin{array}{ll}\text { Density } & \rho_{\text {ref }}=\frac{p_{\text {ref }}}{R T_{r e f}} \sim 1.16 \mathrm{~kg} \mathrm{~m}^{-3} \\ \text { Horizontal velocity } & u_{r e f}=\frac{\tan \phi}{\pi / 2} \frac{N^{2}}{f_{0}^{2}} \beta h_{s c}^{2} \sim 10 \mathrm{~m} \mathrm{~s}^{-1} \\ \text { Vertical velocity } & w_{r e f}=\frac{h_{s c}}{L_{s y n}} u_{r e f} \sim 0.1 \mathrm{~m} \mathrm{~s}^{-1} \\ \text { Horizontal distance } & L_{s y n}=\frac{N}{f_{0}} h_{s c} \sim 1200 \mathrm{~km} \\ \text { Vertical distance } & h_{s c}=\frac{p_{r e f}}{g \rho_{r e f}} \sim 8.8 \mathrm{~km} \\ \text { Time } & t_{r e f}=\frac{L_{s y n}}{u_{r e f}} \sim 1.2 \times 10^{5} \mathrm{~s}\end{array}$

TABLE 2. Derived reference values for non-dimensionalization of (2.1)

non-dimensionalization,

$$
\left.\begin{array}{rl}
p^{*}=\frac{p}{p_{r e f}}, \quad \rho^{*}=\frac{\rho}{\rho_{r e f}}, \quad \boldsymbol{u}^{*}=\frac{\boldsymbol{u}}{u_{r e f}}, & w^{*}=\frac{w}{u_{r e f}} \frac{c_{1} L_{s y n}}{h_{s c}}, \\
t^{*}=\frac{t u_{r e f}}{c_{1} L_{s y n}}, \quad \boldsymbol{x}^{*}=\frac{\boldsymbol{x}}{c_{1} L_{s y n}}, \quad z^{*}=\frac{z}{h_{s c}}
\end{array}\right\}
$$

Here $u_{r e f}$ is an estimate of the thermal wind shear, $\boldsymbol{x}=\boldsymbol{i} x+\boldsymbol{j} y$, and $c_{1}$ is a constant chosen later to eliminate inessential free parameters from the dimensionless equations.

The Brunt-Väisälä frequency, $N=\sqrt{(g / \bar{\Theta})(\mathrm{d} \bar{\Theta} / \mathrm{d} z)}$, with the overbar denoting a horizontal average, characterizes the vertical variation, $\Delta \Theta \sim T_{r e f}\left(N^{2} h_{s c} / g\right)$, of the potential temperature. An appropriate non-dimensionalization of $\Theta$ then reads

$$
\Theta^{*}=\frac{\Theta}{T_{r e f}}=1+c_{2} \frac{\Delta \Theta}{T_{r e f}}\left(\Theta_{1}\left(z^{*}\right)+\widetilde{\Theta}\right) \quad\left(c_{2}=O(1) \text { to be determined below }\right)
$$

In deriving dimensionless equations, we introduce the Mach and Froude numbers, the dimensionless Coriolis $\beta$-parameter, the Rossby number, the aspect ratio, $\ell$, and the Damköhler number,

$$
\begin{gathered}
M a=\frac{u_{r e f}}{\sqrt{R T_{r e f}}} \approx 3.4 \times 10^{-2}, \quad F r=\frac{u_{r e f}}{N h_{s c}} \approx 1.1 \times 10^{-1}, \quad \beta^{*}=\frac{\beta L_{s y n}}{f_{0}}, \\
R o=\frac{u_{r e f}}{f_{0} L_{s y n}} \approx 1.1 \times 10^{-1}, \quad \ell=\frac{h_{s c}}{L_{s y n}} \approx 7.3 \times 10^{-3}, \quad D a=Q_{\Theta} r e f \frac{t_{r e f}}{T_{r e f}}
\end{gathered}
$$


Note that $\beta^{*} \equiv(\pi / 2) F r / \tan \phi$. The first five of these parameters are then replaced through a set of distinguished asymptotic limits that are in line with Pedlosky's small-Rossby-number derivation of the QG model (see Pedlosky 1987; Klein 2010),

$$
c_{2} \frac{M a^{2}}{F r^{2}}=c_{2} \frac{\Delta \Theta}{T_{r e f}}=\varepsilon, \quad \frac{1}{M a^{2}}=\frac{1}{\varepsilon^{3}}, \quad \frac{1}{R o}=\frac{\widehat{f}_{0}}{\varepsilon}, \quad \beta^{*}=\widehat{\beta} \varepsilon, \quad \frac{c_{1}}{\ell}=\frac{1}{\varepsilon^{2}},
$$

where $\left(\widehat{f_{0}}, \widehat{\beta}\right)=O(1)$ as $\varepsilon \rightarrow 0$. The constants $c_{1}, c_{2}$ are included in these distinguished limits in hindsight in such a way that they cancel from the dimensionless equations (2.7) below. For the scaling of the Damköhler number, $D a$, see (4.13) below.

Dropping the ${ }^{*}$ and ${ }^{\wedge}$ indicators for clarity, we obtain the dimensionless equations

$$
\begin{gathered}
\frac{\partial \boldsymbol{u}}{\partial t}+\boldsymbol{u} \cdot \nabla_{\|} \boldsymbol{u}+w \frac{\partial \boldsymbol{u}}{\partial z}+\frac{1}{\varepsilon^{3}} \frac{1}{\rho} \nabla_{\|} p+\frac{1}{\varepsilon}\left(f_{0}+\varepsilon \beta y\right) \boldsymbol{k} \times \boldsymbol{u}=0, \\
\frac{\partial w}{\partial t}+\boldsymbol{u} \cdot \nabla_{\|} w+w \frac{\partial w}{\partial z}+\frac{1}{\varepsilon^{5}} \frac{1}{\rho} \frac{\partial p}{\partial z}=-\frac{1}{\varepsilon^{5}} \\
\frac{\partial \rho}{\partial t}+\boldsymbol{u} \cdot \nabla_{\|} \rho+w \frac{\partial \rho}{\partial z}+\rho \nabla_{\|} \cdot \boldsymbol{u}+\rho \frac{\partial w}{\partial z}=0 \\
\frac{\partial \widetilde{\Theta}}{\partial t}+\boldsymbol{u} \cdot \nabla_{\|} \widetilde{\Theta}+w \frac{\partial \Theta}{\partial z}+w \frac{\mathrm{d} \Theta_{1}}{\mathrm{~d} z}=\frac{D a}{\varepsilon} Q_{\Theta}, \\
\varepsilon\left(\widetilde{\Theta}+\Theta_{1}\right)=\frac{p^{1 / \gamma}}{\rho}-1 .
\end{gathered}
$$

For the reader familiar with Klein's work, we remark that the atmospheric flow parameters in table 1 are essentially equivalent to those used in the general modelling framework in (Klein 2010), while the reference quantities for non-dimensionalization in table 2 have been constructed from these to fit the present application and streamline the subsequent developments.

\section{Quasi-geostrophic far-field flow}

This section describes the asymptotic expansions and the key results for the far field including its separation into a smooth and a singular vortex-induced part.

\subsection{Expansion scheme for the $Q G$ far-field flow}

Here we rederive the QG theory following (Pedlosky 1987) except for one modification: while the classical expansions proceed in integer powers of $\varepsilon$, we allow intermediate level perturbations of $O\left(\varepsilon^{i+1 / 2}\right)$ for integers $i$. The latter appear because the synoptic and vortex core scales differ by $O\left(\varepsilon^{1 / 2}\right)$ (see $\S 4.1 .1$ ) and they are needed for consistently matched asymptotic solutions. For simplicity we neglect diabatic effects in the outer flow. The analysis then proceeds with the expansion scheme

$$
\begin{gathered}
p=p_{0}+\varepsilon p_{1}+\varepsilon^{2}\left(p^{(2)}+p_{2}\right)+\varepsilon^{5 / 2}\left(p^{(5 / 2)}+p_{5 / 2}\right)+o\left(\varepsilon^{5 / 2}\right), \\
\rho=\rho_{0}+\varepsilon \rho_{1}+\varepsilon^{2}\left(\rho^{(2)}+\rho_{2}\right)+\varepsilon^{5 / 2}\left(\rho^{(5 / 2)}+\rho_{5 / 2}\right)+o\left(\varepsilon^{5 / 2}\right) \\
\Theta=1+\varepsilon \Theta_{1}+\varepsilon^{2}\left(\Theta^{(2)}+\Theta_{2}\right)+\varepsilon^{5 / 2}\left(\Theta^{(5 / 2)}+\Theta_{5 / 2}\right)+o\left(\varepsilon^{5 / 2}\right), \\
\boldsymbol{u}=\boldsymbol{u}^{(0)}+\varepsilon^{1 / 2} \boldsymbol{u}^{(1 / 2)}+\varepsilon \boldsymbol{u}^{(1)}+\varepsilon^{3 / 2} \boldsymbol{u}^{(3 / 2)}+o\left(\varepsilon^{3 / 2}\right) \\
w=\varepsilon w^{(1)}+\varepsilon^{3 / 2} w^{(3 / 2)}+o\left(\varepsilon^{3 / 2}\right)
\end{gathered}
$$


where $\left(p_{i}, \rho_{i}, \Theta_{i}\right)(z)$ and $\left(\boldsymbol{u}^{(i)}, w^{(i)}, p^{(i)}, \rho^{(i)}, \Theta^{(i)}\right)(t, \boldsymbol{x}, z)$ represent the mean background state and the flow variability, respectively.

From the scalings of the pressure gradient and Coriolis terms in the horizontal momentum balance from $(2.7 a)$ it follows that, under leading-order geostrophic balance, deviations of the pressure from the background state will arise first at $O\left(\varepsilon^{2}\right)$. The vertical momentum equation in $(2.7 b)$ is dominated by hydrostatic balance up to at least five orders in $\varepsilon$ and, as a consequence, deviations of density from the background state follow the pressure scaling and also start at $O\left(\varepsilon^{2}\right)$. This explains the expansion schemes in $(3.1 a)-(3.1 c)$. In (3.1e) we have used that the vertical velocity in a QG flow is smaller by one order in the Rossby number than expected on the basis of the aspect ratio scaling because the leading-order horizontal divergence vanishes under geostrophic balance (see e.g. Pedlosky 1987).

To streamline the notation, we let

$$
\delta \equiv \varepsilon^{1 / 2}
$$

from here on and use the following abbreviations in the rest of this section:

$$
\left(\begin{array}{c}
\boldsymbol{u} \\
\widetilde{\boldsymbol{u}} \\
w \\
\pi \\
\widetilde{\Theta}
\end{array}\right) \equiv\left(\begin{array}{c}
\boldsymbol{u}^{(0)}+\delta \boldsymbol{u}^{(1 / 2)} \\
\boldsymbol{u}^{(1)}+\delta \boldsymbol{u}^{(3 / 2)} \\
w^{(1)}+\delta w^{(3 / 2)} \\
\left(p^{(2)}+\delta p^{(5 / 2)}\right) / \rho_{0} \\
\Theta^{(2)}+\delta \Theta^{(5 / 2)}
\end{array}\right) .
$$

\subsection{QG theory}

Using (3.3), the combined leading- and first-order results in $\delta$ from the momentum equations, the mass balance, and the potential temperature transport equation read

geostrophic balance

$$
f_{0} \boldsymbol{k} \times \boldsymbol{u}+\nabla_{\|} \pi=0
$$

hydrostatic balance

$$
\frac{\partial \pi}{\partial z}=\widetilde{\Theta}
$$

anelastic constraint

$$
\rho_{0} \nabla_{\|} \cdot \widetilde{\boldsymbol{u}}+\frac{\partial}{\partial z}\left(\rho_{0} w\right)=0,
$$

potential temperature transport

$$
\frac{\partial \widetilde{\Theta}}{\partial t}+\boldsymbol{u} \cdot \nabla_{\|} \widetilde{\Theta}+w \frac{\mathrm{d} \Theta_{1}}{\mathrm{~d} z}=0 .
$$

For later reference, we conclude from (3.4a) that

$$
\nabla_{\|} \cdot \boldsymbol{u}=0 \text { and } \boldsymbol{u}=-\boldsymbol{k} \times \nabla_{\|} \psi,
$$

where the streamfunction, $\psi$, for the horizontal flow is defined as

$$
\psi \equiv-\pi / f_{0}
$$

An additional equation for the divergence of the perturbation velocity, $\nabla_{\|} \cdot \widetilde{\boldsymbol{u}}$, appearing in $(3.4 c)$ is obtained from the curl of the next-order horizontal momentum equation, 
vorticity transport

$$
\left(\frac{\partial}{\partial t}+\boldsymbol{u} \cdot \nabla_{\|}\right)(\zeta+\beta y)+f_{0} \nabla_{\|} \cdot \widetilde{\boldsymbol{u}}=0,
$$

with the relative vertical vorticity

$$
\zeta=k \cdot\left(\nabla_{\|} \times u\right) .
$$

Equations (3.4)-(3.8) are the quasi-geostrophic model for the unknowns $(\boldsymbol{u}, w, \pi, \widetilde{\Theta}$, $\left.\left[\nabla_{\|} \cdot \widetilde{\boldsymbol{u}}\right]\right)(t, \boldsymbol{x}, z)$ defined in (3.3) given the background state through $\rho_{0}(z)$ and $\left(\mathrm{d} \Theta_{1} / \mathrm{d} z\right)(z)$. The essence of the system is revealed through its classical formulation involving an advection equation

$$
\left(\frac{\partial}{\partial t}+\boldsymbol{u} \cdot \nabla_{\|}\right) q=0
$$

for the QG potential vorticity

$$
q=\zeta+\beta y+\frac{f_{0}}{\rho_{0}} \frac{\partial}{\partial z}\left(\frac{\rho_{0}}{\Theta_{1}^{\prime}} \widetilde{\Theta}\right) \quad \text { where } \Theta_{1}^{\prime}=\frac{\mathrm{d} \Theta_{1}}{\mathrm{~d} z} .
$$

To verify these equations, one eliminates $\left[\nabla_{\|} \cdot \tilde{\boldsymbol{u}}\right]$ from (3.7) using (3.4c), and then eliminates $w$ from the remaining equation using (3.4d).

Using that $\zeta=-\nabla_{\|}^{2} \psi$ due to (3.8), (3.6), (3.5), and the geostrophic balance from (3.4a), equation (3.10) becomes an elliptic equation for $\psi$ given the potential vorticity, namely

$$
\left(\nabla_{\|}^{2}+\mathscr{L}_{z}\right) \psi=-q_{r e l},
$$

where

$$
\mathscr{L}_{z}[\cdot]=\frac{f_{0}^{2}}{\rho_{0}} \frac{\partial}{\partial z}\left(\frac{\rho_{0}}{\Theta_{1}^{\prime}} \frac{\partial \cdot}{\partial z}\right) \quad \text { and } \quad q_{r e l}=q-\beta y .
$$

\subsection{Singular vortex theory}

\subsubsection{Regular-singular far-field flow decomposition}

Consider the relative potential vorticity, $q_{r e l}$, to be composed of a line singularity, $q_{s}$, located on the vortex centreline, and a regular contribution, $q_{r}$, so that

$$
q_{r e l}=q_{s}+q_{r}
$$

where

$$
\begin{aligned}
q_{s}(t, \boldsymbol{x}, z) & =\int_{0}^{H} \Gamma\left(t, z^{\prime}\right) \delta_{3}\left([\boldsymbol{x}+\boldsymbol{k} z]-\left[\boldsymbol{X}\left(t, z^{\prime}\right)+\boldsymbol{k} z^{\prime}\right]\right) \mathrm{d} z^{\prime} \\
& =\Gamma(t, z) \delta_{2}(\boldsymbol{x}-\boldsymbol{X}(t, z)) .
\end{aligned}
$$

Here $\delta_{k}(\cdot)$ denote the $k$-dimensional delta distributions, and

$$
\begin{gathered}
\Gamma(t, z)=\Gamma^{(0)}(t, z)+\delta \Gamma^{(1)}(t, z)+o(\delta), \\
\boldsymbol{X}(t, z)=\boldsymbol{X}_{0}(t)+\delta \boldsymbol{X}^{(1)}(t, z)+o(\delta),
\end{gathered}
$$

are the total vortex circulation and the horizontal position of its centreline, respectively. 
To construct a perturbation expansion of $\psi$ that explicitly represents the local QG flow response to the vortex singularity we first rewrite (3.11) as

$$
\left(\nabla_{\|}^{2}+\mathscr{L}_{z}\right) \psi=-q_{s}-q_{r}
$$

Next, introducing local polar coordinates via

$$
\boldsymbol{x}-\boldsymbol{X}(t, z)=r \boldsymbol{e}_{r}=r(\boldsymbol{i} \cos \theta+\boldsymbol{j} \sin \theta),
$$

with $\boldsymbol{e}_{r}$ a radial, and $\boldsymbol{i}, \boldsymbol{j}$ Cartesian horizontal unit vectors, and observing that the quasi-two-dimensional potential vortex streamfunction

$$
\psi_{0}^{2 d}(t, r, z)=-\frac{\Gamma(t, z)}{2 \pi} \ln r \quad \text { solves } \nabla_{\|}^{2} \psi_{0}^{2 d}=-q_{s}
$$

we let, in a first step,

$$
\psi(t, \boldsymbol{x}, z)=\psi_{0}^{2 d}(t, r, z)+\psi_{1}^{*}(t, \boldsymbol{x}, z)
$$

Then, $\psi_{1}^{*}$ obviously satisfies

$$
\left(\nabla_{\|}^{2}+\mathscr{L}_{z}\right) \psi_{1}^{*}=-\mathscr{L}_{z} \psi_{0}^{2 d}-q_{r}
$$

Taking into account the expansion of the centreline in $(3.15 b)$ and the coordinate transformation in (3.17) we express the first term on the right of this equation as

$$
-\mathscr{L}_{z} \psi_{0}^{2 d}=4 M_{0}(t, z) \ln r+\frac{\delta}{r} 2 M_{1}(t, \theta, z)+\frac{\delta^{2}}{r^{2}} 4 M_{2}(t, \theta, z),
$$

where

$$
\begin{gathered}
M_{0}=\frac{1}{8 \pi} \frac{f_{0}^{2}}{\rho_{0}} \frac{\partial}{\partial z}\left(\frac{\rho_{0}}{\Theta_{1}^{\prime}} \frac{\partial \Gamma}{\partial z}\right), \\
M_{1}=-\frac{f_{0}^{2}}{4 \pi \rho_{0} \Gamma} \frac{\partial}{\partial z}\left(\frac{\rho_{0} \Gamma^{2}}{\Theta_{1}^{\prime}} \frac{\partial \boldsymbol{X}^{(1)}}{\partial z}\right) \cdot \boldsymbol{e}_{r} \equiv-\boldsymbol{M}_{1} \cdot \boldsymbol{e}_{r}, \\
M_{2}=\frac{f_{0}^{2} \Gamma}{8 \pi \Theta_{1}^{\prime}}\left(\left[\boldsymbol{e}_{\theta} \cdot \frac{\partial \boldsymbol{X}^{(1)}}{\partial z}\right]^{2}-\left[\boldsymbol{e}_{r} \cdot \frac{\partial \boldsymbol{X}^{(1)}}{\partial z}\right]^{2}\right) .
\end{gathered}
$$

The source term in (3.20) is still singular, yet the singularity is much weaker than the original Dirac distribution in (3.14). Also, the singular part of this source term as given in (3.21) involves the first and second circumferential Fourier modes through the terms $M_{1}(t, \theta, z)$ and $M_{2}(t, \theta, z)$, respectively. For later reference we extract again the dominant singular behaviour of the streamfunction by introducing

$$
\psi_{1}^{2 d}=M_{0} r^{2}(\ln r-1)+\delta M_{1} r \ln r-\delta^{2} M_{2},
$$

which solves

$$
\nabla_{\|}^{2} \psi_{1}^{2 d}=-\mathscr{L}_{z} \psi_{0}^{2 d}(t, r, z)
$$

Collecting results we have

$$
\psi=-\frac{\Gamma}{2 \pi} \ln r+M_{0} r^{2}(\ln r-1)+\delta M_{1} r \ln r-\delta^{2} M_{2}+\psi_{2}^{*},
$$

where the regularized streamfunction, $\psi_{2}^{*}$, satisfies

$$
\left(\nabla_{\|}^{2}+\mathscr{L}_{z}\right) \psi_{2}^{*}=-\mathscr{L}_{z} \psi_{1}^{2 d}-q_{r} .
$$


With (3.25) we have an explicit representation of the singular behaviour of the streamfunction near the vortex centreline $(r \rightarrow 0)$, including its expansion in terms of $\delta$. In appendix B we discuss the behaviour of $\psi_{2}^{*}$ at $r=0$ and inter alia show that it is continuous.

\section{Vortex core structure}

\subsection{Expansion scheme for the mesoscale vortex core flow in gradient wind balance}

\subsubsection{Pressure, velocity, and spatial scaling}

If a given total vortex circulation, $\Gamma$, is realized on the synoptic scale with velocites of order $u_{r e f}$, i.e. $\Gamma=O\left(2 \pi L_{s y n} u_{r e f}\right)$, then it is realized with wind speeds of order $u_{\text {ref }} / \delta$ on the smaller meso scale $L_{m e s}=O\left(\delta L_{s y n}\right)$. At the same time, if the synopticscale Rossby number is $R o=u_{\text {ref }} / f_{0} L_{s y n}=O\left(\delta^{2}\right)$ (see (2.6)) then the vortex Rossby number becomes $R o_{\text {mes }}=u_{\text {mes }} / f_{0} L_{\text {mes }}=O(1)$ and the vortex is in approximate gradient wind balance (see $\S 1.2$ ). This sets the length, velocity and pressure scales for the vortex core,

$$
L_{m e s}=\delta L_{s y n}, \quad \Delta p_{m e s}=O\left(\delta^{4} p_{\text {ref }}\right) \equiv O\left(\varepsilon^{2} p_{\text {ref }}\right), \quad u_{\text {mes }}=O\left(\delta^{-1} u_{\text {ref }}\right) .
$$

Given that $u_{\text {ref }} \sim 10 \mathrm{~m} \mathrm{~s}^{-1}$ (see table 2) and $\varepsilon \sim 0.1$ or $\delta=\sqrt{\varepsilon} \sim 1 / 3$ (see (2.4), (2.6)) these scalings are compatible with tropical storms and nascent hurricanes, which feature velocities of $O\left(30 \mathrm{~m} \mathrm{~s}^{-1}\right)$. Motivated by changes of storm intensities being observed over a day or two $\left(t_{\text {ref }} \sim 30 \mathrm{~h}\right.$ in table 2$)$, we assume the core structure to evolve on the synoptic time scale, i.e. on the same time scale as the quasi-geostrophic outer flow.

\subsubsection{Co-moving coordinate system}

For the meso-scale analysis we introduce vortex-centred horizontal coordinates so that

$$
\boldsymbol{x}=\boldsymbol{X}(t, z)+\delta \widehat{\boldsymbol{x}}=\boldsymbol{X}_{0}(t)+\delta\left(\boldsymbol{X}^{(1)}(t, z)+\widehat{\boldsymbol{x}}\right) .
$$

The relative horizontal offset, $\widehat{x}$, resolves the vortex core diameter scale as estimated in (4.1). To efficiently deal with the near-axisymmetry of the vortex we use the polar coordinates from (3.17) with an appropriate rescaling of the radius, i.e.

$$
\widehat{\boldsymbol{x}}=\widehat{x} \boldsymbol{i}+\widehat{y} \boldsymbol{j} \quad \text { where } \begin{cases}\widehat{x}=\widehat{r} \cos \theta ; & \boldsymbol{i}=\boldsymbol{e}_{r} \cos \theta-\boldsymbol{e}_{\theta} \sin \theta, \\ \widehat{y}=\widehat{r} \sin \theta ; & \boldsymbol{j}=\boldsymbol{e}_{r} \sin \theta+\boldsymbol{e}_{\theta} \cos \theta,\end{cases}
$$

with $\boldsymbol{e}_{r}$ and $\boldsymbol{e}_{\theta}$ the radial and circumferential unit vectors, respectively. For later reference, the polar coordinate representation of the vortex tilt is

$$
\boldsymbol{X}^{(1)}=\left(X^{(1)} \cos \theta+Y^{(1)} \sin \theta\right) \boldsymbol{e}_{r}+\left(-X^{(1)} \sin \theta+Y^{(1)} \cos \theta\right) \boldsymbol{e}_{\theta} .
$$

The horizontal velocity is decomposed into the vortex motion plus the relative velocity,

$$
\boldsymbol{u}=\frac{\partial \boldsymbol{X}}{\partial t}+\left(u_{r} \boldsymbol{e}_{r}+u_{\theta} \boldsymbol{e}_{\theta}\right)
$$

and the transformation rules for derivatives now read

$$
\begin{gathered}
\nabla_{\|}=\delta^{-1}\left(\boldsymbol{e}_{r} \frac{\partial}{\partial \widehat{r}}+\boldsymbol{e}_{\theta} \frac{1}{\widehat{r}} \frac{\partial}{\partial \theta}\right) \equiv \delta^{-1} \widehat{\nabla}, \\
\left.\frac{\partial}{\partial z}\right|_{t, x, y}=\left.\frac{\partial}{\partial z}\right|_{t, \widehat{r}, \theta}-\delta^{-1} \frac{\partial \boldsymbol{X}}{\partial z} \cdot \widehat{\nabla},
\end{gathered}
$$




$$
\left.\frac{\partial}{\partial t}\right|_{x, y, z}=\left.\frac{\partial}{\partial t}\right|_{\widehat{r}, \theta, z}-\delta^{-1} \boldsymbol{V} \cdot \widehat{\nabla} .
$$

For later reference our notation for the Fourier expansion of functions of $\theta$ is

$$
F(\theta)=F_{0}+\sum_{n}\left(F_{n 1} \sin (n \theta)+F_{n 2} \cos (n \theta)\right) .
$$

\subsubsection{Vortex core expansion scheme}

Up to $O(1)$ the circumferential velocity is assumed to be axisymmetric, so that

$$
\begin{aligned}
u_{\theta}(t, \boldsymbol{x}, z ; \varepsilon)= & \delta^{-1} u_{\theta}^{(0)}(t, \widehat{r}, z)+u_{\theta}^{(1)}(t, \widehat{r}, z)+\delta u_{\theta}^{(2)}(t, \widehat{r}, \theta, z)+o(\delta), \\
& u_{r}(t, \boldsymbol{x}, z ; \varepsilon)=\delta u_{r}^{(2)}(t, \widehat{r}, \theta, z)+o(\delta) .
\end{aligned}
$$

That the first-order velocity must be axisymmetric can be derived instead of having to be assumed (Mikusky 2007) (E. Päschke's maiden name). Asymmetries of $O\left(\delta u_{r e f}\right)$ in the horizontal velocity distributed over the core size length scale, $L_{\text {mes }}$, induce horizontal divergences of order $\delta u_{r e f} / L_{m e s}=u_{r e f} / L_{s y n}$, see (4.1). The flow field is essentially anelastic as we will see below, and from the anelastic divergence constraint follow dimensionless vertical velocities of order $w / u_{r e f}=O\left(h_{s c} / L_{s y n}\right)$, and this implies, given the vertical velocity scaling in (2.2),

$$
w(t, \boldsymbol{x}, z ; \varepsilon)=\widehat{w}^{(1)}(t, \widehat{r}, \theta, z)+o(1) .
$$

Expansions for the thermodynamic variables are anticipated as follows: the estimates in (4.1) settle the lowest order at which horizontal pressure variations can occur. All lower-order pressure terms will be horizontally homogeneous. Moreover, they will have to match the corresponding terms in the outer QG solution since any differences would be equilibrated rapidly by fast internal waves. The outer pressure expansion does not include half-order scalings below $\varepsilon^{2} \equiv \delta^{4}$, and this is why such terms must also be absent in the core. The pressure perturbations turn out to be hydrostatic in the regime considered so that half-order scalings cannot appear in the $\Theta$ and $\rho$ expansion at the respective orders either. These considerations lead to

$$
\begin{gathered}
p=p_{0}+\delta^{2} p_{1}+\delta^{4}\left(\widehat{p}^{(4)}+\widehat{p}_{4}\right)+\delta^{5}\left(\widehat{p}^{(5)}+\widehat{p}_{5}\right)+o\left(\delta^{5}\right), \\
\rho=\rho_{0}+\delta^{2} \rho_{1}+\delta^{4}\left(\widehat{\rho}^{(4)}+\widehat{\rho}_{4}\right)+\delta^{5}\left(\widehat{\rho}^{(5)}+\widehat{\rho}_{5}\right)+o\left(\delta^{5}\right), \\
\Theta=1+\delta^{2} \Theta_{1}+\delta^{4}\left(\widehat{\Theta}^{(4)}+\widehat{\Theta}_{4}\right)+\delta^{5}\left(\widehat{\Theta}^{(5)}+\widehat{\Theta}_{5}\right)+o\left(\delta^{5}\right) .
\end{gathered}
$$

Here $\left(p_{0}, p_{1}, \rho_{0}, \rho_{1}, \Theta_{1}\right)$ represent the background state as in $(3.1),\left(\widehat{p}_{i}, \widehat{\rho}_{i}, \widehat{\Theta}_{i}\right)(t, z)$ are higher-order horizontal mean values, and $\left(\widehat{p}^{(i)}, \widehat{\rho}^{(i)}, \widehat{\Theta}^{(i)}\right)(t, \widehat{r}, \theta, z)$ are the perturbation variables of interest in this section. With the diabatic source terms scaling as

$$
\frac{D a}{\varepsilon} Q_{\Theta}=\delta^{2} Q_{\Theta}^{(2)}+o\left(\delta^{5}\right)
$$

they will modify the adiabatic dynamics but will not change the balances fundamentally (see discussion below (4.19)). This also settles the asymptotic scaling of the Damköhler number as announced in $§ 2$ (below (2.6)).

\subsection{Asymptotic equation hierarchy for the vortex core}

The governing equations transformed to the co-moving coordinates are provided in appendix A. Inserting the vortex core expansion scheme from $\S 4.1 .3$ we obtain horizontal momentum balance 
$O\left(\delta^{-3}\right)$

$$
\begin{gathered}
-\frac{\left(u_{\theta}^{(0)}\right)^{2}}{\widehat{r}}+\frac{1}{\rho_{0}} \frac{\partial \widehat{p}^{(4)}}{\partial \widehat{r}}-f_{0} u_{\theta}^{(0)}=0 \\
\frac{\partial \widehat{p}^{(4)}}{\partial \theta}=0
\end{gathered}
$$

$O\left(\delta^{-2}\right)$

$$
\begin{gathered}
-\frac{2 u_{\theta}^{(0)} u_{\theta}^{(1)}}{\widehat{r}}+\frac{1}{\rho_{0}} \frac{\partial \widehat{p}^{(5)}}{\partial \widehat{r}}-f_{0}\left(u_{\theta}^{(1)}+\boldsymbol{e}_{\theta} \cdot \frac{\mathrm{d} \boldsymbol{X}_{0}}{\mathrm{~d} t}\right)=0 \\
\frac{1}{\rho_{0} \widehat{r}} \frac{\partial \widehat{p}^{(5)}}{\partial \theta}+f_{0} \boldsymbol{e}_{r} \cdot \frac{\mathrm{d} \boldsymbol{X}_{0}}{\mathrm{~d} t}=0
\end{gathered}
$$

$O\left(\delta^{-1}\right)$

$$
\begin{aligned}
& \frac{u_{\theta}^{(0)}}{\widehat{r}} \frac{\partial u_{r}^{(2)}}{\partial \theta}-\frac{2 u_{\theta}^{(0)} u_{\theta}^{(2)}}{\widehat{r}}-\frac{\left(u_{\theta}^{(1)}\right)^{2}}{\widehat{r}}+\boldsymbol{e}_{\theta} \cdot \frac{\partial \boldsymbol{X}^{(1)}}{\partial z} \frac{\widehat{w}^{(1)} u_{\theta}^{(0)}}{\widehat{r}} \\
& \quad+\frac{1}{\rho_{0}} \frac{\partial \widehat{p}^{(6)}}{\partial \widehat{r}}-\frac{\rho_{1}}{\rho_{0}^{2}} \frac{\partial \widehat{p}^{(4)}}{\partial \widehat{r}}-f_{0}\left(u_{\theta}^{(2)}+\boldsymbol{e}_{\theta} \cdot \frac{\partial \boldsymbol{X}^{(1)}}{\partial t}\right)-\beta Y_{C}^{(0)} u_{\theta}^{(0)}=0 \\
& \frac{\partial u_{\theta}^{(0)}}{\partial t}+\widehat{w}^{(1)} \frac{\partial u_{\theta}^{(0)}}{\partial z}+u_{r}^{(2)}\left(\frac{\partial u_{\theta}^{(0)}}{\partial \widehat{r}}+\frac{u_{\theta}^{(0)}}{\widehat{r}}\right)+\frac{u_{\theta}^{(0)}}{\widehat{r}} \frac{\partial u_{\theta}^{(2)}}{\partial \theta} \\
& \quad-\widehat{w}^{(1)} \boldsymbol{e}_{r} \cdot \frac{\partial \boldsymbol{X}^{(1)}}{\partial z} \frac{\partial u_{\theta}^{(0)}}{\partial \widehat{r}}+\frac{1}{\rho_{0} \widehat{r}} \frac{\partial \widehat{p}^{(6)}}{\partial \theta}+f_{0}\left(u_{r}^{(2)}+\boldsymbol{e}_{r} \cdot \frac{\partial \boldsymbol{X}^{(1)}}{\partial t}\right)=0
\end{aligned}
$$

The flow is hydrostatic to very high order with the first non-trivial vertical balance vertical momentum balance $O\left(\delta^{-6}\right)$ :

$$
\frac{\partial \widehat{p}^{(4)}}{\partial z}-\frac{\partial \boldsymbol{X}^{(1)}}{\partial z} \cdot \hat{\nabla}_{\|} \widehat{p}^{(4)}=-\widehat{\rho}^{(4)}
$$

The leading two contributions to the flow velocity are horizontal and axisymmetric according to (4.10), (4.11), and therefore divergence-free. For the second-order velocity field we find an anelastic divergence constraint that is modified by the vortex tilt, mass continuity

$O(1)$ :

$$
\frac{\rho_{0}}{\widehat{r}}\left(\frac{\partial}{\partial \widehat{r}}\left(\widehat{r} u_{r}^{(2)}\right)+\frac{\partial u_{\theta}^{(2)}}{\partial \theta}\right)+\frac{\partial}{\partial z}\left(\rho_{0} \widehat{w}^{(1)}\right)-\frac{\partial \boldsymbol{X}^{(1)}}{\partial z} \cdot \hat{\nabla}_{\|}\left(\rho_{0} \widehat{w}^{(1)}\right)=0 .
$$

The first non-trivial potential temperature transport equation reads potential temperature $O\left(\delta^{2}\right)$ :

$$
\frac{u_{\theta}^{(0)}}{\widehat{r}} \frac{\partial \widehat{\Theta}^{(4)}}{\partial \theta}+\widehat{w}^{(1)} \frac{\mathrm{d} \Theta_{1}}{\mathrm{~d} z}=Q_{\Theta}^{(2)} .
$$

For any $\widehat{w}^{(i)}$ with $i<1$ we would have obtained $\widehat{w}^{(i)} \mathrm{d} \Theta_{1} / \mathrm{d} z=Q_{\Theta}^{(i+1)}$, so that these orders of $w$ would be induced solely by diabatic sources. As a consequence, the 
lowest-order vertical velocity that remains in the diabatic case is $\widehat{w}^{(1)}$ as anticipated in the expansion scheme (4.11). Stronger diabatic effects will be considered in future work.

The equation of state relates the perturbations of the thermodynamic variables through

equation of state

$O\left(\delta^{4}\right)$ :

$$
\widehat{\rho}^{(4)}=\rho_{0}\left(\frac{\widehat{p}^{(4)}}{\gamma p_{0}}-\widehat{\Theta}^{(4)}\right)
$$

\subsection{Temporal evolution of the vortex structure}

The goal here is to derive from (4.14)-(4.20) an evolution equation for the leadingorder circumferential velocity, $u_{\theta}^{(0)}$, given the vortex tilt, $\partial \boldsymbol{X}^{(1)} / \partial z$, and the diabatic source term, $Q_{\Theta}^{(2)}$. Consider to this end the second-order circumferential momentum balance from (4.16b). Averaging in $\theta$ taking into account that $u_{\theta}^{(0)}$ is axisymmetric we obtain

$$
\frac{\partial u_{\theta}^{(0)}}{\partial t}+\widehat{w}_{0}^{(1)} \frac{\partial u_{\theta}^{(0)}}{\partial z}+u_{r, 0}^{(2)}\left(\frac{\partial u_{\theta}^{(0)}}{\partial \widehat{r}}+\frac{u_{\theta}^{(0)}}{\widehat{r}}+f_{0}\right)-u_{r, *}^{(2)} \frac{\partial u_{\theta}^{(0)}}{\partial \widehat{r}}=0
$$

where, using Fourier decomposition (4.9),

$$
u_{r, *}^{(2)}=\left(\widehat{w}^{(1)} \boldsymbol{e}_{r} \cdot \frac{\partial \boldsymbol{X}^{(1)}}{\partial z}\right)_{0}=\frac{1}{2}\left[\widehat{w}_{12}^{(1)} \frac{\partial X^{(1)}}{\partial z}+\widehat{w}_{11}^{(1)} \frac{\partial Y^{(1)}}{\partial z}\right] .
$$

Expressions for $\widehat{w}_{0}^{(1)}$ and $\widehat{w}_{11}^{(1)}, \widehat{w}_{12}^{(1)}$ follow from the Fourier decomposition of the potential temperature transport equation in (4.19), namely

$$
\begin{gathered}
\widehat{w}_{0}^{(1)} \frac{\mathrm{d} \Theta_{1}}{\mathrm{~d} z}=Q_{\Theta, 0}^{(2)}, \\
\widehat{w}_{1 k}^{(1)} \frac{\mathrm{d} \Theta_{1}}{\mathrm{~d} z}=Q_{\Theta, 1 k}^{(2)}+(-1)^{k} \frac{u_{\theta}^{(0)}}{\widehat{r}} \widehat{\Theta}_{1 k^{*}}^{(4)} \quad\left(k \in\{1,2\}, k^{*}=3-k\right) .
\end{gathered}
$$

Now we have $\widehat{\Theta}_{1 k}^{(4)}=-\widehat{\rho}_{1 k}^{(4)} / \rho_{0}$ from the equation of state, (4.20), because $\widehat{p}^{(4)}$ is axisymmetric according to $(4.14 b)$, and hence $\widehat{p}_{1 k}^{(4)} \equiv 0$. By extracting the first Fourier modes of the vertical momentum balance in (4.17) we find

$$
\widehat{\Theta}_{11}^{(4)}=-\frac{\widehat{\rho}_{11}^{(4)}}{\rho_{0}}=-\frac{1}{\rho_{0}} \frac{\partial Y^{(1)}}{\partial z} \frac{\partial \widehat{p}^{(4)}}{\partial \widehat{r}}, \quad \widehat{\Theta}_{12}^{(4)}=-\frac{\widehat{\rho}_{12}^{(4)}}{\rho_{0}}=-\frac{1}{\rho_{0}} \frac{\partial X^{(1)}}{\partial z} \frac{\partial \widehat{p}^{(4)}}{\partial \widehat{r}} .
$$

Using the gradient wind balance, $(4.14 a)$, to eliminate $\partial \widehat{p}^{(4)} / \partial \widehat{r}$, and going back to (4.23b) we obtain the desired expressions for the $\widehat{w}_{1 k}^{(1)}$ in terms of $u_{\theta}^{(0)}, \partial \boldsymbol{X}^{(1)} / \partial z$, and $Q_{\Theta}^{(2)}$,

$$
\widehat{w}_{1 k}^{(1)}=\frac{1}{\Theta_{1}^{\prime}}\left[Q_{\Theta, 1 k}^{(2)}+(-1)^{k} \frac{\partial X_{k}^{(1)}}{\partial z} \frac{u_{\theta}^{(0)}}{\widehat{r}}\left(\frac{\left(u_{\theta}^{(0)}\right)^{2}}{\widehat{r}}+f_{0} u_{\theta}^{(0)}\right)\right] \quad(k=1,2),
$$

where $X_{1}^{(1)} \equiv X^{(1)}$ and $X_{2}^{(1)} \equiv Y^{(1)}$. Inserting into (4.22) we find the expression for $u_{r, *}^{(2)}$ (called $u_{r, *}$ there for simplicity of notation) given in $(1.3 d)$. 
To find a corresponding expression for $u_{r, 0}^{(2)}$ (see the third term in (4.21)), consider the circumferential average of mass continuity, (4.18). A brief calculation yields first

$$
\frac{\partial\left(\widehat{r} u_{r, 0}^{(2)}\right)}{\partial \widehat{r}}=-\frac{\widehat{r}}{\rho_{0}} \frac{\partial\left(\rho_{0} \widehat{w}_{0}^{(1)}\right)}{\partial z}+\frac{1}{2}\left[\frac{\partial X^{(1)}}{\partial z} \frac{\partial\left(\widehat{r} \widehat{w}_{12}^{(1)}\right)}{\partial \widehat{r}}+\frac{\partial Y^{(1)}}{\partial z} \frac{\partial\left(\widehat{r} \widehat{w}_{11}^{(1)}\right)}{\partial \widehat{r}}\right] .
$$

Then, exploiting (4.23) and (4.25), we integrate in $\widehat{r}$ requiring that $u_{r, 0}^{(2)}$ be finite at $r=0$ to find

$$
u_{r, 0}^{(2)}=-\frac{1}{\widehat{r}} \int_{0}^{\widehat{r}} \frac{r^{\prime}}{\rho_{0}} \frac{\partial}{\partial z}\left(\rho_{0} \frac{Q_{0}^{(2)}}{\Theta_{1}^{\prime}}\right) \mathrm{d} r^{\prime}+u_{r, *}^{(2)} \equiv u_{r, 00}^{(2)}+u_{r, *}^{(2)} .
$$

Equations (4.21)-(4.27) now yield the core structure evolution equation given in $(1.3 a)$.

\subsection{First Fourier modes of the second-order horizontal flow}

Here we study the second-order horizontal momentum and mass balances in (4.16) and (4.18) to derive expressions for the first $\theta$-Fourier modes of $u_{r}^{(2)}, u_{\theta}^{(2)}$. These will be of particular interest in matching the inner and outer solutions in $\S 5$ as their large- $\widehat{r}$ behaviour determines the evolution of the vortex centreline perturbation, $\boldsymbol{X}^{(1)}$.

The non-symmetric components of $u_{r}^{(2)}$ and $u_{\theta}^{(2)}$ are represented by a velocity potential, $\phi^{(2)}$ (divergent part) and a streamfunction, $\psi^{(2)}$ (non-divergent part) so that

$$
u_{r}^{(2)}=\frac{1}{\widehat{r}} \frac{\partial \psi^{(2)}}{\partial \theta}+\frac{\partial \phi^{(2)}}{\partial \widehat{r}}, \quad u_{\theta}^{(2)}=-\frac{\partial \psi^{(2)}}{\partial \widehat{r}}+\frac{1}{\widehat{r}} \frac{\partial \phi^{(2)}}{\partial \theta} .
$$

After projection onto the first Fourier modes, mass continuity (4.18) yields

$$
\left(\frac{\partial^{2}}{\partial \widehat{r}^{2}}+\frac{1}{\widehat{\widehat{r}}} \frac{\partial}{\partial \widehat{r}}-\frac{1}{\widehat{r}^{2}}\right) \phi_{1 k}^{(2)}=-\frac{1}{\rho_{0}} \frac{\partial\left(\rho_{0} \widehat{w}_{1 k}^{(1)}\right)}{\partial z}+\frac{1}{2}\left(\boldsymbol{e}_{r} \cdot \frac{\partial \boldsymbol{X}^{(1)}}{\partial z}\right)_{k} \frac{\partial \widehat{w}_{0}^{(1)}}{\partial \widehat{r}} \equiv R_{1 k}
$$

Knowing that the quasi-geostrophic outer flow is horizontally non-divergent we let

$$
\phi_{1 k}^{(2)}=-\widehat{r} \int_{\widehat{r}}^{\infty} \frac{1}{\bar{r}^{3}}\left[\int_{0}^{\bar{r}} \overline{\bar{r}}^{2} R_{1 k} \mathrm{~d} \overline{\bar{r}}\right] \mathrm{d} \bar{r} .
$$

The integral in (4.30) converges and yields a vanishing divergent part of the secondorder flow as $\widehat{r} \rightarrow \infty$ provided $\widehat{w}_{0}^{(1)}$ and $\widehat{w}_{1 k}^{(1)}$ decay sufficiently rapidly for large $\widehat{r}$. Considering (4.23a) and (4.25), this is the case if (i) $Q_{\Theta, 0}^{(2)}$ and $Q_{\Theta, 1 k}^{(2)}$ decay sufficiently rapidly, and (ii) $u_{\theta}^{(0)}=\Gamma /(2 \pi \widehat{r})(1+o(1))$ as $\widehat{r} \rightarrow \infty$. For the diabatic source terms we take the liberty of assuming such rapid decay, while the required large- $\widehat{r}$ behaviour of $u_{\theta}^{(0)}$ follows from our assumption that the vortex has a well-defined total circulation, $\Gamma(t, z)$.

With the divergent part of the flow vanishing for large $\widehat{r}$, the solutions for the streamfunction $\psi^{(2)}$ will be central in determining the evolution of the vortex tilt. Elimination of $\widehat{p}^{(4)}$ and $\widehat{p}^{(6)}$ from $(4.16 a)$ and $(4.16 b)$ by cross-differentiation, extraction of the first Fourier modes from the resulting equations, and inserting (4.28) yields

$$
\left(\frac{\partial^{2}}{\partial \widehat{r}^{2}}+\frac{1}{\widehat{r}} \frac{\partial}{\partial \widehat{r}}-\left[\frac{\zeta_{\hat{r}}^{(0)}}{u_{\theta}^{(0)}}+\frac{1}{\widehat{r}^{2}}\right]\right) \psi_{1 k}^{(2)}=\frac{(-1)^{k^{*}}}{u_{\theta}^{(0)}}(\mathscr{H}+\mathscr{I}+\mathscr{J}+\mathscr{Q})_{1 k^{*}},
$$


where $k^{*}=3-k$,

$$
\zeta^{(0)}=\frac{\partial u_{\theta}^{(0)}}{\partial \widehat{r}}+\frac{u_{\theta}^{(0)}}{\widehat{r}}
$$

is the vorticity of the leading-order axisymmetric circumferential flow, and

$$
\begin{gathered}
\mathscr{H}_{1 k}=\frac{\partial}{\partial \widehat{r}}\left(\widehat{r} \widehat{w}_{1 k}^{(1)} \frac{\partial u_{\theta}^{(0)}}{\partial z}\right), \\
\mathscr{I}_{1 k}=-\widehat{r}\left[\zeta^{(0)}+f_{0}\right] \frac{1}{\rho_{0}} \frac{\partial\left(\rho_{0} \widehat{w}_{1 k}^{(1)}\right)}{\partial z}, \\
\mathscr{J}_{1 k}=\frac{\partial \phi_{1 k}^{(2)}}{\partial \widehat{r}}\left(\widehat{r} \frac{\partial \zeta^{(0)}}{\partial \widehat{r}}\right), \\
\mathscr{Q}_{1 k}=\left(\boldsymbol{e}_{r} \cdot \frac{\partial \boldsymbol{X}^{(1)}}{\partial z}\right)_{k}\left(\widehat{w}_{0}^{(1)} \frac{u_{\theta}^{(0)}}{\widehat{r}}-\frac{\partial}{\partial r}\left(\widehat{r} \widehat{w}_{0}^{(1)} \frac{\partial u_{\theta}^{(0)}}{\partial r}\right)\right) .
\end{gathered}
$$

Particular solutions with homogeneous boundary conditions

$$
\psi_{1 k}^{(2)}=\frac{\partial \psi_{1 k}^{(2)}}{\partial \widehat{r}}=0 \quad \text { at } \widehat{r}=0
$$

are

$$
\psi_{1 k}^{(2)}=(-1)^{k^{*}} \widehat{r}\left[\frac{u}{\widehat{r}} \int_{0}^{\widehat{r}} \frac{1}{\bar{r} u^{2}} \int_{0}^{\bar{r}} r^{\prime}[\mathscr{H}+\mathscr{I}+\mathscr{J}+\mathscr{Q}]_{1 k^{*}}\left(r^{\prime}\right) \mathrm{d} r^{\prime} \mathrm{d} \bar{r}\right] .
$$

This is also the final solution because one of the two linearly independent homogeneous solutions produces singular velocities near $\widehat{r}$; the other represents a singular dipole flow for large $\widehat{r}$ which is incompatible with the outer QG flow (see $\S$ C.1 for details).

We have restricted this section to those results from the core structure analysis that are needed to derive our main results, i.e. the core structure evolution equation (see $\S 4.3$ ), and the evolution equation for the vortex tilt (see $\S 5$ below). For more details of the core structure solution, such as the higher harmonics of $\widehat{p}^{(4)}, \widehat{\rho}^{(4)}, \widehat{\Theta}^{(4)}, \widehat{w}^{(1)}$, an evaluation of the first-order horizontal momentum balances in (4.15), and the higher harmonics of $\phi^{(2)}$ and $\psi^{(2)}$ see appendix C.

\section{Matching and the vortex centreline velocity}

\subsection{Eckhaus' notation for the matching procedure}

Here we employ Van Dyke's procedure for the asymptotic matching of the far-field and vortex core solutions (Van Dyke 1964) using Eckhaus' notation (Eckhaus 1979): let $\Phi_{\delta}(x)$ be defined for $x \in D \subset \mathbb{R}^{n}$ and $0<\delta \ll 1$. When $\Phi_{\delta}$ solves a boundarylayer-type problem, there is one subdomain $D_{0} \subset D$ within which $\Phi_{\delta}$ has a regular expansion

$$
\left.\begin{array}{rl}
\Phi_{\delta}(x) & =\sum_{n=0}^{m} \delta^{n} \Phi^{(n)}(x)+o\left(\delta^{m}\right) \\
& \equiv\left(E^{(m)} \Phi_{\delta}\right)(x)+o\left(\delta^{m}\right)
\end{array}\right\} \quad \text { for } x \in D_{0},
$$


and another subset $S_{\delta} \subset D$ such that $\Phi_{\delta}$ when expressed in boundary layer coordinates,

$$
\Phi_{\delta}(x) \equiv \widehat{\Phi}_{\delta}(\widehat{x}) \quad \text { where } \widehat{x}=x / \delta,
$$

has an expansion

$$
\left.\begin{array}{rl}
\widehat{\Phi}_{\delta}(\widehat{x}) & =\sum_{n=m_{0}}^{m} \delta^{n} \widehat{\Phi}^{(n)}(\widehat{x})+o\left(\delta^{m}\right) \\
& \equiv\left(\widehat{E}^{(m)} \widehat{\Phi}_{\delta}\right)(\widehat{x})+o\left(\delta^{m}\right)
\end{array}\right\} \quad \text { for } \widehat{x}=\widehat{S} .
$$

Here $\widehat{x} \in \widehat{S}$ for some $\delta$-independent $\widehat{S} \subset \mathbb{R}^{n}$ implies $x \in S_{\delta}$. In boundary-layer-type problems the size of $S_{\delta}$ shrinks to zero as $\delta \rightarrow 0$. In (5.1), (5.3), $E^{(m)}$ is the $(m+1)$ term expansion of functions of $x$ in $D_{0}$, while $\widehat{E}^{(m)}$ is the expansion of functions of $\widehat{x}$ in $\widehat{S}$; note that $\widehat{E}^{(m)}$ allows a different scaling of the leading-order term than $E^{(m)}$.

Loosely speaking, Van Dyke's matching principle requires 'the near-field behaviour of the outer solution to match the far-field behaviour of the inner solution' when both are expressed in the inner coordinates. Let $T$ denote the transformation from (5.2), i.e.

$$
\left(T \Phi_{\delta}\right)(\widehat{x}) \equiv \widehat{\Phi}_{\delta}(\widehat{x})=\Phi_{\delta}\left(T^{-1}(\widehat{x})\right) \quad \text { with } T x=\widehat{x}=x / \delta .
$$

Then, given the expansion depth $m_{o}$ of the outer expansion, the matching principle reads

$$
\widehat{E}^{\left(m_{i}\right)} T E^{\left(m_{o}\right)} \Phi_{\delta}=\widehat{E}^{\left(m_{i}\right)} T E^{\left(m_{o}\right)} T^{-1} \widehat{E}^{\left(m_{i}\right)} T \Phi_{\delta}
$$

for the smallest possible inner expansion depth $m_{i}$. Part of the matching procedure is to determine values for $m_{o}$ and $m_{i}$ such that the resulting system of equations for the asymptotic inner and outer representations of $\Phi_{\delta}$ is closed and sufficiently accurate.

This simplified description of the matching principle suffices for the present purposes (for a more comprehensive exposition of the technique, see Eckhaus 1979).

\subsection{The centreline equation of motion}

Matching the horizontal velocities in this section will yield the equation of motion for the vortex centreline in analogy with the analysis of three-dimensional slender vortex filaments (see Ting et al. 2007, and references therein). For the horizontal velocities, we keep terms up to and including $O(\delta)$, so that $m_{o}=1$ in (5.5).

\subsubsection{The near-field behaviour of the outer solution}

Based on (3.25) and recalling that $\psi_{2}^{*}$ represents the non-singular part of the outer flow, we find the near-field behaviour of the horizontal velocity in the outer solution

$$
\begin{aligned}
\widehat{E}^{(1)} T E^{(1)} \boldsymbol{u}= & \boldsymbol{u}_{0}^{(0)}+\delta\left(\boldsymbol{u}_{0}^{(1 / 2)}+\boldsymbol{X}^{(1)} \cdot\left(\nabla_{\|} \boldsymbol{u}\right)_{0}^{(0)}\right) \\
& +\left(\frac{1}{\delta} \frac{\Gamma}{2 \pi \widehat{r}}-\delta M_{0} \widehat{r}(2 \ln \widehat{r}-1+2 \ln \delta)\right) \boldsymbol{e}_{\theta}+\delta\left(\widehat{r} \boldsymbol{e}_{r} \cdot\left(\nabla_{\|} \boldsymbol{u}\right)_{0}^{(0)}\right) \\
& +\delta\left(\left[\ln \widehat{r}-\ln \frac{1}{\delta}\right] \boldsymbol{k} \times \boldsymbol{M}_{1}+\left(\boldsymbol{M}_{1} \cdot \boldsymbol{e}_{r}\right) \boldsymbol{e}_{\theta}\right),
\end{aligned}
$$

where $\boldsymbol{u}_{0}$ and $\left(\boldsymbol{\nabla}_{\|} \boldsymbol{u}\right)_{0}$ denote the regular part of the outer flow velocity and its horizontal gradient evaluated at $\left(t, \boldsymbol{X}_{0}, z\right)$, and $M_{0}$ and $\boldsymbol{M}_{1}$ were defined in (3.22). 
We arrive at this result by (i) calculating $-\boldsymbol{k} \times \nabla_{\|} \psi_{s}$ from the outer solution in (3.25), and (ii) taking into account that $\psi_{2}^{*}$ is sufficiently smooth up to $O(\delta)$ (see appendix B) so that the associated velocity has a two-term Taylor expansion near the centreline and becomes a contribution to the regular part of the outer velocity, $\boldsymbol{u}_{0}$.

For later reference, we note that $\widehat{\boldsymbol{r}} \boldsymbol{e}_{r} \cdot\left(\nabla_{\|} \boldsymbol{u}\right)_{0}^{(0)}$ in (5.6) can be expressed in terms of a local quadratic streamfunction, and the local vorticity, $\zeta_{0}^{*}$ (see appendix D)

$$
\widehat{\boldsymbol{r}}_{r} \cdot\left(\boldsymbol{\nabla}_{\|} \boldsymbol{u}\right)_{0}^{(0)}=\widehat{r} \zeta_{0}^{*} \boldsymbol{e}_{\theta}-\boldsymbol{k} \times \hat{\boldsymbol{\nabla}}_{\|}\left(\widehat{r}^{2}\left(\psi_{21}^{*} \sin (2 \theta)+\psi_{22}^{*} \cos (2 \theta)\right)\right),
$$

with constants $\psi_{2 k}^{*}$ for $k \in\{1,2\}$.

\subsubsection{The far-field behaviour of the inner solution}

We recall from (4.5), (3.15b), (4.10), and (4.28) that the absolute horizontal velocity within the vortex core up to and including terms of $O(\delta)$ is

$$
\begin{aligned}
\widehat{E}^{(1)} T \boldsymbol{u}= & \frac{\mathrm{d} \boldsymbol{X}_{0}}{\mathrm{~d} t}+\delta \frac{\partial \boldsymbol{X}^{(1)}}{\partial t}+\frac{1}{\delta}\left(u_{\theta}^{(0)}+\delta u_{\theta}^{(1)}+\delta^{2} u_{\theta, 0}^{(2)}\right) \boldsymbol{e}_{\theta} \\
& +\delta\left(\nabla_{\|} \phi^{(2)}-\boldsymbol{k} \times \nabla_{\|} \psi^{(2)}\right) .
\end{aligned}
$$

For the divergent part of the flow we have, by construction of $\phi^{(2)}$ in (4.30), that

$$
E^{(1)} T^{-1}\left[\nabla_{\|} \phi^{(2)}\right]=0
$$

For the far-field behaviour of $-\boldsymbol{k} \times \nabla_{\|} \psi^{(2)}$ in (5.8), consider the expression for $\psi_{1 k}^{(2)}$ in (4.35). With de L'Hôspital's rule for limits of type ' $\infty / \infty$ ', the term in brackets obeys

$$
\lim _{r \rightarrow \infty}\left[\frac{u}{\widehat{r}} \int_{0}^{\widehat{r}} \frac{1}{\bar{r} u^{2}} \int_{0}^{\bar{r}} r^{\prime} \mathscr{F}\left(r^{\prime}\right) \mathrm{d} r^{\prime} \mathrm{d} \bar{r}\right]=\frac{\pi}{\Gamma} \int_{0}^{\infty} r \mathscr{F}(r) \mathrm{d} r \equiv L[\mathscr{F}],
$$

provided $\left[u, \widehat{r}^{2} \mathrm{~d} u / \mathrm{d} \widehat{r}\right]=\left[\Gamma / 2 \pi \widehat{r},-\Gamma / 2 \pi \widehat{r}^{2}\right](1+o(1))$ as $\widehat{r} \rightarrow \infty$, and the integral exists.

The integral converges for $\mathscr{F} \in\left\{\mathscr{H}_{1 k}, \mathscr{J}_{1 k}\right\}$ (see (4.33), (4.35)), and it does for $\mathscr{F}=\mathscr{Q}_{1 k}$ if the diabatic source term vanishes sufficiently rapidly for large $\widehat{r}$ (see appendix E). For $\mathscr{F}=\mathscr{I}_{1 k}$, extraction of its dominant part and application of L'Hôspital's rules reveals a logarithmic divergence for large $\widehat{r}$ (see appendix E). Combining all results we find

$$
\widehat{E}^{(1)} T E^{(1)} T^{-1}\left[\tilde{\psi}_{1}^{(2)}\right]=\left(-\widehat{r} \ln \widehat{r} \boldsymbol{M}_{1}(\tau, z)+\widehat{r} \Psi(\tau, z)\right) \cdot \boldsymbol{e}_{r}(\theta),
$$

where $\tilde{\psi}_{1}^{(2)}=\psi_{11}^{(2)} \sin \theta+\psi_{12}^{(2)} \cos \theta$,

$$
\Psi=L[\mathcal{H}+\widetilde{\mathcal{I}}+\mathcal{J}+\mathcal{Q}]
$$

with $\mathcal{K}=-\boldsymbol{i} \mathscr{K}_{11}+\boldsymbol{j} \mathscr{K}_{12}$ for $\mathscr{K} \in\{\mathscr{H}, \widetilde{I}, \mathscr{J}, \mathscr{Q}\}$ and $L[\cdot]$ defined in (5.10). Here

$$
\widetilde{\mathscr{I}_{1 k}}=\mathscr{I}_{1 k}+H(\widehat{r}-1) I_{1 k} / \widehat{r}^{2}
$$

is the integrable part of $\mathscr{I}_{1 k}$ in that $\int_{0}^{\infty} r \widetilde{\mathscr{I}_{1 k}} \mathrm{~d} r$ exists, $H(\cdot)$ is the Heaviside step function, and $I_{1 k}$ is defined by $\boldsymbol{M}_{1}=(\pi / \Gamma)\left(-\boldsymbol{i} I_{11}+\boldsymbol{j} I_{12}\right)$ with $\boldsymbol{M}_{1}$ from $(3.22 b)$. 
Combining (5.8), (5.11), we find the far-field behaviour of the inner horizontal velocity,

$$
\begin{aligned}
\widehat{E}^{(1)} T E^{(1)} T^{-1} \widehat{E}^{(1)} T \boldsymbol{u}= & \frac{\mathrm{d} \boldsymbol{X}_{0}}{\mathrm{~d} t}+\delta \frac{\partial \boldsymbol{X}^{(1)}}{\partial t} \\
& +\widehat{E}^{(1)} T E^{(1)} T^{-1}\left[\frac{1}{\delta}\left(u_{\theta}^{(0)}+\delta u_{\theta}^{(1)}+\delta^{2} u_{\theta, 0}^{(2)}\right)\right] \boldsymbol{e}_{\theta} \\
& -\widehat{E}^{(1)} T E^{(1)} T^{-1}\left[\delta \boldsymbol{k} \times \widehat{\nabla} \tilde{\psi}_{2}^{(2)}\right] \\
& +\delta\left(\ln \widehat{r} \boldsymbol{k} \times \boldsymbol{M}_{1}+\left(\boldsymbol{M}_{1} \cdot \boldsymbol{e}_{r}\right) \boldsymbol{e}_{\theta}-\boldsymbol{k} \times \boldsymbol{\Psi}\right),
\end{aligned}
$$

where $\tilde{\psi}_{2}^{(2)}=\psi_{21}^{(2)} \sin (2 \theta)+\psi_{22}^{(2)} \cos (2 \theta)$.

\subsubsection{Matching}

Here we match the large- $\widehat{r}$ inner solution in (5.14) with the near-field outer solution in (5.6). Comparing the second line in (5.14) with the second line in (5.6) taking into account (5.7) we find for the axisymmetric contribution

$$
\left.\begin{array}{l}
u_{\theta}^{(0)}=\frac{\Gamma^{(0)}}{2 \pi \widehat{r}}(1+o(1)) \\
u_{\theta}^{(1)}=\frac{\Gamma^{(1 / 2)}}{2 \pi \widehat{r}}(1+o(1)) \\
u_{\theta, 0}^{(2)}=\widehat{r}\left(\zeta_{0}^{*}-M_{0}(2 \ln \widehat{r}-1+2 \ln \delta)\right)(1+o(1))
\end{array}\right\} \quad \text { as } \widehat{r} \rightarrow \infty .
$$

The results for $u_{\theta}^{(0)}, u_{\theta}^{(1)}$ were expected from the theory of point vortices, while $u_{\theta}^{(2)}$ involves an additional influence of baroclinicity, $\partial \Gamma / \partial z$, through $M_{0}$ (see (3.22)).

The respective asymmetric contribution of these terms in (5.6) according to (5.7) and the third line of (5.14) provide the large- $\widehat{r}$ behaviour of the Fourier modes, $\psi_{2 k}^{(1)}$, of the perturbation streamfunction in the vortex core,

$$
\widehat{E}^{(1)} T E^{(1)} T^{-1} \psi_{2 k}^{(1)}=r^{2} \psi_{2 k}^{*}(1+o(1)) \quad \text { as } r \rightarrow \infty .
$$

On one hand, the $\psi_{2 k}^{*}$ are related to the irrotational strain of the regular part of the outer solution in $\boldsymbol{X}_{0}$ (see appendix D). On the other hand, they represent the homogeneous solutions to the equations for the second Fourier modes of the stream function of the core flow which are thus determined (see the discussion before (C 12) and (C 13) in appendix C).

Comparing the remaining terms in (5.6) and (5.14) we find the centreline velocity,

$$
\begin{gathered}
\frac{\mathrm{d} \boldsymbol{X}_{0}}{\mathrm{~d} t}=\boldsymbol{u}_{0}^{(0)}, \\
\frac{\partial \boldsymbol{X}^{(1)}}{\partial t}=\boldsymbol{u}_{0}^{(1 / 2)}+\boldsymbol{X}^{(1)} \cdot\left(\nabla_{\|} \boldsymbol{u}\right)_{0}^{(0)}-\ln \frac{1}{\delta}\left(\boldsymbol{k} \times \boldsymbol{M}_{1}\right)+(\boldsymbol{k} \times \boldsymbol{\Psi}) .
\end{gathered}
$$

\section{Vortex Rossby waves (VRWs) and quasi-modes}

\subsection{VRWs and quasi-modes in the literature}

In a series of papers, Montgomery and colleagues discuss the roles of VRW damping and the long-lived precession of so-called quasi-modes in stabilizing an atmospheric vortex against vertical shear of the background flow (see Shapiro \& Montgomery 1993; Montgomery \& Kallenbach 1997; Reasor \& Montgomery 2001; McWilliams et al. 2003; Reasor et al. 2004, and references therein). VRWs are analogous to the classical 
mid-latitude Rossby waves, except that in VRWs meridional advection of planetary rotation is replaced with radial advection of the axisymmetric mean vorticity of the vortex. Quasi-modes arise in these linear theories as the characteristic flow patterns associated with the low-frequency continuous spectrum of the linearized perturbation operators. Here we discuss why VRW dynamics on the vortex core cannot be captured by the present asymptotic theory whereas it does provide a nonlinear theory for the quasi-modes. Note, however, that VRWs in the outer flow are captured as part of the QG outer solution.

If a vortex features a long-range decay of vorticity away from its core, then a critical radial layer may exist within which circumferential advection by the mean vortex and the phase propagation of VRWs coincide and this resonance induces wave damping. Of particular interest in the present context are VRWs of circumferential Fourier mode one as these may be interpreted as oscillatory lateral displacements of the entire vortex and thus correspond roughly with vortex centreline motions. Why do VRWs (Montgomery \& Kallenbach 1997) on the vortex core and the associated damping mechanism not emerge from the present asymptotic analysis? This is understood by comparing the characteristic time scales. The circumferential phase speed of VRWs is comparable with the circumferential advection time scale of the mean vortex, $T_{V R W} \sim L_{\text {mes }} / u_{\max }$, where $L_{\text {mes }}$ is the radial location of the maximum circumferential mean velocity, $u_{\max }$. In contrast, the vortex centreline motions analysed in this paper develop on longer time scales of order $L_{s y n} / u_{r e f}=\delta^{-2} T_{V R W}$. In the language of matched asymptotics for concentrated vortices, the VRWs thus correspond to the fast-time solutions discussed by Ling \& Ting (1988) (see also Ting et al. 2007), whereas the present centreline equations of motion correspond to slow-time solutions.

Reasor et al. (2004), studying dry flow dynamics, find that vortex Rossby wave damping is weak in the typical flow regime for hurricane development. This is demonstrated through numerical simulations based on the non-hydrostatic primitive equations for initially upright barotropic vortices with Gaussian vorticity distribution and maximum circumferential mean velocities of $u_{\max }=10 \ldots 40 \mathrm{~m} \mathrm{~s}^{-1}$ at a radius of $L_{\text {mes }}=100 \mathrm{~km}$ corresponding with vortex Rossby numbers $R o_{\text {mes }}=u_{\text {max }} / f_{0} L_{\text {mes }}=$ $3.2 \ldots 12.7$. The internal Rossby radii of the background state are $L_{s y n}=N H / \pi f_{0}=$ $630 \ldots 1250 \mathrm{~km}$, so the vortices are concentrated with $L_{\text {mes }} / L_{\text {syn }} \sim 0.08 \ldots 0.16$. The stability of the background stratification is characterized by $N / f_{0}=\pi L_{s y n} / H=$ $200 \ldots 400$, where $N$ is the (constant) Brunt-Väisälä frequency, and $H=10 \mathrm{~km}$ is the height of the flow domain. The stabilizing vortex Rossby wave damping is weak in these cases for two reasons: (i) the critical radius lies far outside the vortex core in a region with very small remaining radial vorticity gradient, and (ii) the vortex turnover time at the critical radius is very long because of the slow remaining circumferential velocity and because of the large length of the circumference. With weak VRW damping, these vortices support long-time precessions of quasi-modes with circumferential Fourier mode one, and these correspond roughly with horizontal displacements of the entire vortex structure as considered here.

Reasor et al. propose a heuristic model to describe these nearly undamped quasimodes motivated by previous linearized analyses of Schecter, Montgomery \& Reasor (2002) and Schecter \& Montgomery (2003) in the context of the quasi-geostrophic and the asymmetric balanced model of Shapiro \& Montgomery (1993). The heuristic model states that potential vorticity perturbations, $q^{\prime}$, due to the precessing quasi-mode 
should obey

$$
q^{\prime}=-\frac{F_{q, s}(r, z)}{\omega_{p}}\left[\sin \left(\lambda-\omega_{p} t\right)-\sin (\lambda)\right],
$$

where $\lambda$ denotes the circumferential angle relative to the background vertical shear direction, $F_{q, s}(r, z)$ is a forcing function that depends on the structure of the unperturbed axisymmetric vortex, and $\omega_{p}$ is the angular frequency of precession.

There are indications in the literature that VRW damping may not be weak at all in moist vortex flows (Schecter \& Montgomery 2007). However, an in-depth discussion of moist flows is beyond the scope of the present paper.

\subsection{Quasi-modes on the vortex core in the present theory}

Here we demonstrate that, for vortices in gradient wind balance, i.e. for vortex Rossby numbers $R o_{\text {mes }}=u_{\text {max }} / f_{0} L_{\text {mes }}=O(1)$, the present theory provides an explanation of such quasi-modes as the net result of the vortex centreline dynamics. The first-order centreline evolution equation in (5.17b) may be rewritten as (see appendix F)

$$
\frac{\partial \boldsymbol{X}^{(1)}}{\partial t}=\boldsymbol{u}_{0}^{(1 / 2)}+\boldsymbol{X}^{(1)} \cdot\left(\nabla_{\|} \boldsymbol{u}\right)_{0}^{(0)}-\boldsymbol{k} \times\left(A \frac{\partial^{2} \boldsymbol{X}^{(1)}}{\partial z^{2}}+B \frac{\partial \boldsymbol{X}^{(1)}}{\partial z}\right)
$$

where

$$
A=\ln \frac{1}{\delta} \frac{\Gamma}{4 \pi} \frac{f_{0}^{2}}{\Theta_{1}^{\prime}}-\tilde{A}, \quad B=\ln \frac{1}{\delta} \frac{\Gamma}{4 \pi} \frac{f_{0}^{2}}{\Theta_{1}^{\prime}} \frac{\partial}{\partial z}\left(\ln \left[\frac{\rho_{0} \Gamma^{2}}{\Theta_{1}^{\prime}}\right]\right)-\tilde{B}
$$

and where $\tilde{A}(t, z), \tilde{B}(t, z)$ are integral expressions involving the leading-order vortex core structure as detailed in appendix F, (F6)-(F 8). In the absence of diabatic forcing, the core structure becomes time independent as discussed in $\S 4.3$, so that all coefficients in (6.2) depend on $z$ only. Following Reasor et al. (2004) in assuming, in addition, a barotropic leading-order vortex and constant (logarithmic) background stratification, corresponding to $\Theta_{1}^{\prime}=$ const, the equations further simplify. Finally, since Reasor et al. (2004) use models based on the Boussinesq approximation for the development of their vortex alignment theory, we may consider here the simplified cases of exponential, $\rho_{0}(z)=\rho_{\infty} \exp \left(-z / H_{\rho}\right)$ or constant, $\rho_{0}(z) \equiv \rho_{\infty}$, background density. In both cases, all coefficients in (6.2) become constant, i.e. independent of $z$.

Reasor et al. (2004) assume forcing of an initially vertically aligned barotropic vortex by horizontally homogeneous easterly vertically sheared winds, so that

$$
\boldsymbol{u}_{0}^{(1 / 2)}=(U \cos (\pi z / H), 0)^{\mathrm{T}},
$$

and $\left(\nabla_{\|} \boldsymbol{u}\right)_{0}^{(0)} \equiv 0$ in (6.2), where $H$ is the height of the top boundary of the flow domain.

At the bottom and top of the domain, they assume constant-potential-temperature boundary conditions which, considering the potential temperature transport equation and the fact that the potential temperature is horizontally homogeneous in the far field, corresponds to rigid lid conditions, i.e. to $w=0$ at $z=0$ and $z=H$. Recalling (4.25) for the first Fourier modes of the vertical velocity in the vortex core and considering the adiabatic case like Reasor et al. (2004), we find that rigid lid conditions require

$$
\frac{\partial \boldsymbol{X}^{(1)}}{\partial z}=0 \text { at } z=0 \text { and } z=H .
$$

This provides the top and bottom boundary conditions for solutions of (6.2). 
With these simplifications, (6.2) is linear in $\boldsymbol{X}^{(1)}$, so that solutions may be constructed as superpositions of a particular solution $\boldsymbol{X}_{p}^{(1)}(z)$ satisfying

$$
A \frac{\mathrm{d}^{2} \boldsymbol{X}_{p}^{(1)}}{\mathrm{d} z^{2}}+B \frac{\mathrm{d} \boldsymbol{X}_{p}^{(1)}}{\mathrm{d} z}=-\boldsymbol{k} \times \boldsymbol{u}_{0}^{(1 / 2)}
$$

and suitable homogeneous solutions which satisfy

$$
\frac{\partial \boldsymbol{X}_{h}^{(1)}}{\partial t}=-\boldsymbol{k} \times\left(A \frac{\partial^{2} \boldsymbol{X}_{h}^{(1)}}{\partial z^{2}}+B \frac{\partial \boldsymbol{X}_{h}^{(1)}}{\partial z}\right)
$$

and will guarantee compliance with the initial conditions.

Particular solutions satisfying (6.6) for $\boldsymbol{u}_{0}^{(1 / 2)}(z)$ from (6.4) are of the form

$$
\boldsymbol{X}_{p}^{(1)}(z)=\left(X_{0}, a \cos (\pi z / H)+b \sin (\pi z / H)+Y_{1} \exp (-B z / A)+Y_{0}\right)^{\mathrm{T}}
$$

where

$$
a=U\left(\frac{A \pi^{2}}{H^{2}}+\frac{B^{2}}{A}\right)^{-1}, \quad b=-\frac{B H}{A \pi} a .
$$

$\boldsymbol{X}_{p}^{(1)}$ satisfies the boundary conditions from (6.5) provided $b=Y_{1}=0$, so that

$$
\boldsymbol{X}_{p}^{(1)}(z)=\left(X_{0}, a \cos (\pi z / H)+Y_{0}\right)^{\mathrm{T}} .
$$

This finding corroborates and extends earlier results in the literature: for a concentrated atmospheric vortex embedded in a background vertical shear there is an equilibrium configuration involving a down-shear left tilt of the vortex centre (see Reasor et al. 2004, and references therein). We extend this in that we allow for horizontal displacements of the vortex centre comparable to the vortex core size whereas earlier studies relied on linearization and small-displacement assumptions.

Real-valued homogeneous eigenmode solutions from (6.7) satisfying the boundary conditions in (6.5) read

$$
\boldsymbol{X}_{h, k}^{(1)}(t, z)=\mathrm{e}^{\mathrm{i} \omega_{k} t} \mathrm{e}^{-\mu z}\left(\mathrm{e}^{\mathrm{i} v_{k} z}-\frac{\mu-\mathrm{i} v_{k}}{\mu+\mathrm{i} v_{k}} \mathrm{e}^{-\mathrm{i} v_{k} z}\right)\left[\boldsymbol{X}_{0}^{(1)}+\mathrm{i} \boldsymbol{k} \times \boldsymbol{X}_{0}^{(1)}\right]+\text { c.c. },
$$

where c.c. indicates the complex conjugate, and

$$
\omega_{k}=\frac{B^{2}}{4 A}+\frac{A \pi^{2}}{H^{2}} k^{2}, \quad \mu=\frac{B}{2 A}, \quad v_{k}=\frac{\pi}{H} k \quad(k=1,2, \ldots) .
$$

For the Boussinesq case, $\rho_{0} \equiv \rho_{\infty}=$ const, we have $\mu=B=0$ and the eigenmode solutions further simplify. In particular, the gravest mode with $k=1$ becomes

$$
\boldsymbol{X}_{h, 1}^{(1)}(t, z)=\cos (\pi z / H)\left(\cos (\omega t) \boldsymbol{X}_{0}^{(1)}+\sin (\omega t) \boldsymbol{k} \times \boldsymbol{X}_{0}^{(1)}\right) .
$$

For an initially upright vortex perturbed by the shear from (6.4) we find

$$
\boldsymbol{X}^{(1)}(t, z)=\frac{U}{\omega} \cos (\pi z / H)\left(\begin{array}{c}
\sin (\omega t) \\
1-\cos (\omega t)
\end{array}\right) \quad \text { where } \omega=\frac{A \pi^{2}}{H^{2}} .
$$

A quantitative comparison with Reasor et al. (2004) reveals that the precession frequency extracted from simulations of vortices with Gaussian distribution of 
potential vorticity agrees well with the present theory for their first case in their $\S 5$. For this case $u_{\text {max }}=10 \mathrm{~m} \mathrm{~s}^{-1}, L_{m e s}=100 \mathrm{~km}, H=10 \mathrm{~km}, L_{s y n}=N H / \pi f_{0}=1250 \mathrm{~km}$, and the vortex Rossby number $R o_{m e s}=u_{\max } / L_{m e s} f_{0}=3.2$. While Reasor et al. report a ratio of the vortex turnover time at the radius of maximum wind speed and the vortex precession time of $\omega^{*} \equiv \omega L_{\text {mes }} / u_{\max }=0.053$, we have agreement with $\omega^{*}=0.059$ on the basis of (6.14), the $\sim 10 \%$ difference being compatible with a higher-order error in $\delta=\sqrt{L_{m e s} / L_{\text {syn }}} \sim 0.09$ for this case.

For the more intense vortex with $R o_{\text {mes }}=12.7$, however, the discrepancy is substantial with $\omega^{*} \approx 0.2$ in (Reasor et al. 2004) and $\omega^{*} \approx 0.5$ from the present theory. Such a deviation is not surprising because the regime of validity of the present theory is the gradient wind regime which, by definition, has $R o_{\text {mes }}=O(1)$ as $\varepsilon \rightarrow 0$. With actual values of $\varepsilon \sim 1 / 8$, the stronger vortex lies outside this regime since $R o_{\text {mes }}=12.7 \sim 1.5 \varepsilon^{-1}$. Another indication that our theory does not apply to this case comes from the asymptotic scaling. We should have $\omega^{*}=O(\varepsilon)$ as $\varepsilon \rightarrow 0$, whereas the precession and vortex turnover times become comparable for the stronger vortex.

\section{Discussion}

Here we provide an additional discussion of the core structure evolution equation (4.21) and the centreline equations of motion (5.17), and we summarize several limitations of the theory together with possible remedies.

\subsection{The centreline equations of motion}

\subsubsection{General properties}

The left-hand side of the leading-order centreline equation of motion, (5.17a), does not depend on $z$. This implies that the regular part of the quasi-geostrophic background flow on the right is barotropic to leading order, and is consistent with results in the literature stating that (nearly) adiabatic vortices cannot sustain a strong horizontal shear (see e.g. Jones 1995, 2000; Reasor \& Montgomery 2001; Reasor et al. 2004, and references therein). It is also consistent with the observation that strong, concentrated atmospheric vortices develop predominantly in the sub-tropics, where the Coriolis effect and thermal wind shear are reduced in comparison with the middle latitudes. We recall, however, from the previous discussions that the vortex even with the assumed weak shear will undergo horizontal displacements comparable to the vortex core size and will thus be strongly tilted. Given the implied weak shear of the background flow, one may surmise that the outer flow could then have been described by a theory simpler than the quasi-geostrophic one in the first place. We leave an exploration of this possibility for future work.

The first two terms on the right in $(5.17 b)$ describe the first-order advection of the vortex centre by the background flow. The remaining terms capture the self-induced vortex motion. The definitions of $\boldsymbol{M}_{1}$ and $\boldsymbol{\Psi}$ from (3.22b), (5.12), together with (4.33), (4.23a), (4.25) and (4.30), reveal that vertical tilt and baroclinicity, asymmetries of the diabatic sources, and the background density stratification interact nonlinearly to produce this deviation of the vortex centre motion from simple advection by the background wind.

\subsubsection{Local induction}

In the special case of a barotropic vortex, $\partial \Gamma / \partial z \equiv 0$, in an atmosphere with constant density, $\mathrm{d} \rho_{0} / \mathrm{d} z \equiv 0$, and constant stratification, $\mathrm{d}^{2} \Theta_{1} / \mathrm{d} z^{2} \equiv 0$, the leading logarithmic term in $(5.17 b)$ is proportional to $-(\Gamma / 4 \pi) \kappa \boldsymbol{b}$ with $\kappa$ the centreline curvature and $\boldsymbol{b}$ its binormal unit vector. To see this, consider the definition of $\boldsymbol{M}_{1}$ 
in (3.22b) and use that $\partial^{2} \boldsymbol{X}^{(1)} / \partial z^{2}=\kappa \boldsymbol{n}+O(\delta)$ and $\boldsymbol{k}=\boldsymbol{\tau}+o(1)$. At a first glance, this result appears to be in line with the theory of slender vortex filaments (Callegari $\&$ Ting 1978), where it emerges as the leading-order term in the centreline equation of motion. Keeping only this leading term one has the classical 'local induction approximation (LIA)' (see also Ricca 1991; Ting et al. 2007, and references therein). There is a discrepancy, however, in the sign of the term. Whereas a three-dimensional filament moves in the direction of the binormal unit vector for positive circulation, a hurricane-like vortex precesses in the opposite direction according to this term.

To obtain a physical interpretation in the present context, consider (3.20)-(3.22b), which provide the expressions for $\boldsymbol{M}_{1}$ and emerge from the analysis of the QG solutions in the immediate vicinity of the singular vortex. The term in question involving $\boldsymbol{M}_{1}$ is part of the first term in (3.20), i.e. $-\mathscr{L}_{z} \psi_{0}^{2 d}$. Recalling the derivations leading to this point, we find that this term embodies the net effect on the streamfunction of bending a potential vortex, represented by $\psi_{0}^{2 d}$, to follow a curved centreline.

Close inspection of the detailed derivation for three-dimensional vortex filaments by Callegari \& Ting (1978) reveals that the corresponding term is the sum of two terms in the asymptotic evaluation of the Biot-Savart kernel which, in analogy with the present situation, provides the far-field behaviour in the immediate vicinity of the singular vortex line. To verify this, see the first equation in Callegari \& Ting (1978, p. 172) and trace back the derivation of the second term on the right. Now, the first term in the slender-filament theory is in agreement with the present result for atmospheric vortices and has an interpretation analogous to that given in the previous paragraph: it represents the local net effect of bending a potential vortex along a curved centreline. This effect is present independently of whether or not the vortex is long-stretched in the axial direction, like the classical slender vortex, or whether it is a flow in a shallow fluid layer, like the atmospheric vortices considered here. In contrast, the second term in the slender-vortex theory, which is twice as large with opposite sign, does not appear here. This term is the result of non-local induction from vorticity located far away along the vortex filament axis when measured in units of the core diameter. This is also why this term is absent here: due to the extreme 'pancake-like' aspect ratio of the atmospheric vortex its axial extent is small compared to the vortex core diameter and the far-field induction effect does not materialize.

\subsection{Vortex core structure}

\subsubsection{The adiabatic lifting mechanism}

Jones $(1995,2000)$ numerically simulates an adiabatic atmospheric vortex in a background shear. After $6 \mathrm{~h}$ she observes and discusses a constant 'relationship between the direction of the vortex tilt and the orientations of the potential temperature perturbation and vertical-velocity pattern'. This relationship corresponds to the tilt-potential temperature asymmetry described in (4.23b) and (4.25) above. In the adiabatic setting, a wavenumber-one pattern of ascent and descent with a phase shift of $90^{\circ}$ relative to the potential temperature pattern is found which is reflected in (4.23b) (note the index shift on the $\Theta$ modes). These vertical velocity asymmetries induce the lowering and raising of $\Theta$ surfaces called 'adiabatic lifting mechanism' by Frank \& Ritchie (1999).

\subsubsection{The vortex core evolution equation}

Whereas the left-hand side of the vortex core structure evolution equation (1.3a) describes advection of circumferential momentum in the meridional plane of the 
vortex, the right-hand side represents nonlinear interactions of vertical velocity asymmetries with the vortex tilt (see definition of $u_{r, *}$ in $(1.3 d)$ as derived in (4.22)). Such an effect has also been discussed by Shapiro \& Montgomery (1993) in the context of linear and weakly nonlinear theories for balanced perturbations of a strong vertically aligned axisymmetric vortex. Collecting all contributing terms we find that $\partial u_{\theta} / \partial t \equiv 0$ in the absence of diabatic heating even when the vortex has a tilt and associated asymmetric vertical velocity fluctuations. In this case, these asymmetries and the tilt have a particular phase relationship (see $\$ 7.2 .1$ ) that annihilates $u_{r, *}$. This annihilation will not occur in general when diabatic effects are active, and in that case a nonlinear coupling of the vortex core structure evolution with the vortex tilt dynamics arises and is mediated by the first circumferential Fourier modes of the asymmetric heating (see (5.12), (4.33), and the relation between the asymmetric vertical velocities, $\widehat{w}_{1 k}^{(1)}$, and the asymmetric heating in (4.23)). Note that these results on the local self-induced motion due to processes in the vortex core will complement any strengthening, weakening, or alignment processes due to vortex Rossby waves in the QG far field (see Montgomery \& Kallenbach 1997; Reasor \& Montgomery 2001; Schecter et al. 2002, and item (b) in $\$ 7.3$ below), and these processes can be captured here through solutions of the regularized outer flow problem in (1.2).

\subsection{Limitations of the present theory and outlook}

The theory presented here is based on a number of simplifying assumptions that limit its range of applications and deserve scrutiny in future research work.

(a) Large Rossby numbers and the inner core of a hurricane: in this paper we have extended asymptotic theories for concentrated quasi-geostrophic vortices to the gradient wind regime, thereby moving from small to moderate vortex Rossby numbers, and covering 'tropical storms' and 'incipient hurricanes'. Mature hurricanes, however, feature large vortex Rossby numbers with their inner cores in approximate cyclostrophic balance, where pressure gradients and centripetal accelerations dominate the Coriolis effect. Describing such a vortex asymptotically may require a three-layer analysis involving a cyclostrophic inner core, a first outer layer in gradient wind balance, and matching to the geostrophic far field. In such a setting, new dynamical effects will arise due to the fact that the vortex turnover time is no longer separated asymptotically from the vortex Rossby and buoyancy wave eigenfrequencies, so that a Rossby-inertia-buoyancy (RIB) wave instability may occur (see Plougonven \& Zeitlin 2002; Schecter \& Montgomery 2004, 2006, and references therein).

(b) Long-range tails of vorticity: we assume here that the leading-order far-field behaviour at every vertical level is consistent with that of a point vortex. Since turbulent friction and vertical momentum transport due to moist convection will couple the vertical layers, this assumption will have to be modified in a theory for cyclones in moist air. With such an extension one can then address the effect of larger-scale far-field tails of potential vorticity. Combining the VRW theory for a QG vortex to describe the outer flow from Schecter et al. (2002) with the present quasi-stationary theory for the vortex core in gradient wind balance appears to be promising in this context.

(c) Fast processes - vortex stabilization: one central assumption adopted here is that the characteristic time scale of all processes considered is commensurate with the synoptic time scale, i.e. with the characteristic time scale of the large-scale motion of the vortex. This precludes the description of internal waves, VRWs, 
and advection on the vortex core, all of which act on much shorter time scales. Following Ting \& Ling (see e.g. 1983) and Ting et al. (see e.g. 2007) one can incorporate such faster processes systematically through multiple-scales asymptotics in time.

One important effect in this context is the resilience against vertical background shear induced by VRW damping (see, e.g. Reasor et al. 2004, and references therein). Note that VRWs on the vortex core have a systematically shorter time scale than those on the QG outer flow, so the present statement does not contradict the related remark on VRWs under item $(b)$ above.

(d) The $\beta$-drift: the reader might be missing an explicit representation of the beta-drift (see e.g. Smith \& Ulrich 1990; Smith 1991; Reznik 1992; Reznik \& Kizner $2007 b$ ) in (5.17), which is the technical term for the self-induced motion of a vortex due to the large-scale transport of the horizontally inhomogeneous planetary rotation. According to Reznik \& Kizner (2007b) the order of magnitude of the $\beta$-drift velocity is $U_{\beta} \sim \beta L_{s y n}^{2}$, where $L_{s y n}$ is the Rossby deformation radius. In non-dimensional terms using our notation from (2.4), (2.6), this means $U_{\beta} / u_{\text {ref }}=\left(\beta L_{\text {syn }} / f_{0}\right)\left(f_{0} L_{\text {syn }} / u_{\text {ref }}\right)=\beta^{*} / R o=\widehat{\beta} / \widehat{f}_{0}=O(1)$. Thus, the $\beta$-drift will contribute at leading order to the vortex motion. As the effect is due, however, to the advection of planetary rotation over distances comparable to the Rossby deformation radius, $L_{s y n}$, it is an effect that appears implicitly in the outer quasi-geostrophic flow as part of $\boldsymbol{u}_{0}^{(0)}$ in $(5.17 a)$. Within the vortex core of size $L_{m e s} \sim \delta L_{s y n}$ the effect merely generates a dimensionless higher-order contribution of order $\beta L_{\text {mes }}^{2} / u_{\text {ref }} \sim O\left(\delta^{2}\right)$, which is why it does not appear in the vortex-coredependent terms $\boldsymbol{M}_{1}, \boldsymbol{\Psi}$ in $(5.17 b)$.

An explicit representation of the development of the $\beta$-drift is given by Reznik and co-workers in Reznik (1992), Reznik \& Grimshaw (2001), Reznik \& Kizner $(2007 a, b)$ who consider the initial value problem of a concentrated vortex placed in an unperturbed QG background flow on the $\beta$-plane. They find that the rearrangement of planetary rotation by the far-field potential vortex generates two large-scale counter-rotating gyres which in turn induce an effective mean flow component in the vicinity of the vortex. This self-induced flow shifts the entire vortex in the westward direction, and this is called the $\beta$-drift. We note that the outer flow description in the present theory in (1.2) includes explicitly the perturbation of the far field by the presence of the vortex through the streamfunction contributions $\psi_{1}^{2 d}$ and $\psi_{1}^{2 d}$, and we expect future solutions of these equations to reproduce the $\beta$-drift as a contribution to the leading-order centreline velocity.

Reznik \& Grimshaw (2001) analyse the evolution of a singular vortex based on the shallow-water equations. In addition to the quasi-geostrophic part of the $\beta$-effects they reveal ageostrophic dynamics on the faster intertia-gravity wave time scale. Capturing these faster-scale processes in the present theory would require introduction of multiple-time-scale asymptotics.

(e) Stronger diabatic source terms and moist processes: preliminary work by Mikusky (2007) (E. Päschke's maiden name) indicates that the strength of diabatic source terms in the presence of moisture can be an order of magnitude larger in $\delta$ than assumed here. The core structure evolution equation (1.3a) with the strength of the source term from $(1.3 d)$ reveals that with such stronger diabatically induced vertical velocities weaker tilts will suffice to drive changes of the vortex core structure on the same time scale. Also, as mentioned already in $\S 6$, the presence 
of moisture can enhance the damping effect of vortex Rossby waves (Schecter \& Montgomery 2007), thus increasing the vortex resilience against vertical shear.

(f) Boundary layer effects: clearly, we have thus far neglected boundary layer effects, while the established literature (see e.g. Emanuel 2003; Smith \& Montgomery 2010) reports that boundary layer processes will have to be included when moist processes are considered, as these feed and partially determine the vortex secondary circulation.

( $g$ ) Logarithmic terms: it is beyond the scope of the present paper to either attempt an 'analysis beyond all orders' following Keller \& Ward (1996) for the logarithmic terms appearing in the asymptotic matching in $\S 5$, or to exploit the intermediate asymptotic ordering induced by the logarithms as done for slender fluid vortices by Klein \& Majda (1991) (see also Ting et al. 2007).

\section{Acknowledgements}

The authors thank the three referees, among them M. T. Montgomery, for their scrutinizing reading and their tough constructive criticism throughout the review stage of this manuscript. The authors also thank Deutsche Forschungsgemeinschaft for their continuing support through Grants PA 1849/1 and KL 611/14. This paper is dedicated to the memory of our friend and colleague Dr A. Z. Owinoh.

\section{Appendix A. Governing equations in the co-moving coordinates}

Transforming (2.7) to the vortex-centred coordinates from $\S 4.1 .2$ using (4.6)-(4.8), $\boldsymbol{U} \equiv \partial \boldsymbol{X} / \partial t, \boldsymbol{u}_{r e l}=u_{r} \boldsymbol{e}_{r}+u_{\theta} \boldsymbol{e}_{\theta}$, and

$$
\nabla_{\|} \equiv \frac{1}{\delta} \widehat{\nabla} \equiv \frac{1}{\delta}\left(\boldsymbol{e}_{r} \frac{\partial}{\partial \widehat{r}}+\boldsymbol{e}_{\theta} \frac{1}{\widehat{r}} \frac{\partial}{\partial \theta}\right) \quad \text { and }\left.\left.\quad \frac{\partial}{\partial z}\right|_{t, \boldsymbol{x}} \equiv \frac{\partial}{\partial z}\right|_{t, \widehat{x}}-\frac{1}{\delta} \frac{\partial \boldsymbol{X}}{\partial z} \cdot \widehat{\nabla}
$$

we find

$$
\begin{gathered}
\frac{\partial\left(\boldsymbol{U}+\boldsymbol{u}_{r e l}\right)}{\partial t}+\frac{1}{\delta} \boldsymbol{u}_{r e l} \cdot \widehat{\nabla} \boldsymbol{u}_{r e l}+w\left[\frac{\partial}{\partial z}-\frac{1}{\delta} \frac{\partial \boldsymbol{X}}{\partial z} \cdot \widehat{\nabla}\right]\left(\boldsymbol{U}+\boldsymbol{u}_{r e l}\right) \\
+\frac{1}{\delta^{7}} \frac{1}{\rho} \widehat{\nabla} p+\frac{1}{\delta^{2}} f \boldsymbol{k} \times\left(\boldsymbol{U}+\boldsymbol{u}_{r e l}\right)=0 \\
\frac{\partial w}{\partial t}+\frac{1}{\delta} \boldsymbol{u}_{r e l} \cdot \widehat{\nabla} w+w\left[\frac{\partial}{\partial z}-\frac{1}{\delta} \frac{\partial \boldsymbol{X}}{\partial z} \cdot \widehat{\nabla}\right] w \\
+\frac{1}{\delta^{10}}\left(\frac{1}{\rho}\left[\frac{\partial}{\partial z}-\frac{1}{\delta} \frac{\partial \boldsymbol{X}}{\partial z} \cdot \widehat{\nabla}\right] p+1\right)=0 \\
\frac{\partial \rho}{\partial t}+\frac{1}{\delta} \widehat{\nabla} \cdot\left(\rho \boldsymbol{u}_{r e l}\right)+\left[\frac{\partial}{\partial z}-\frac{1}{\delta} \frac{\partial \boldsymbol{X}}{\partial z} \cdot \widehat{\nabla}\right](\rho w)=0 \\
\frac{\partial \widehat{\Theta}}{\partial t}+\frac{1}{\delta} \boldsymbol{u}_{r e l} \cdot \widehat{\nabla} \widehat{\Theta}+w\left[\frac{\partial}{\partial z}-\frac{1}{\delta} w \frac{\partial \boldsymbol{X}}{\partial z} \cdot \widehat{\nabla}\right] \widehat{\Theta}+w \frac{\mathrm{d} \Theta_{1}}{\mathrm{~d} z}=\frac{D a}{\delta^{2}} Q_{\Theta},
\end{gathered}
$$

where $1+\delta^{2}\left(\Theta_{1}(z)+\widetilde{\Theta}\right)=p^{1 / \gamma} / \rho$ and $f=f_{0}+\delta^{2} \beta y$.

\section{Appendix B. The regular part of the outer-flow streamfunction}

To show that under (3.26) $\psi_{2}^{*}$ will be continuous at $r=0$, consider a repetition of the prodedure from $\S 3.3 .1$. Let $\psi_{2}^{2 d}$ solve

$$
\nabla_{\|}^{2} \psi_{2}^{2 d}=-\mathscr{L}_{z} \psi_{1}^{2 d}
$$


Applying $\mathscr{L}_{z}$ to (3.23) the most singular behaviour in $r$ is generated by the second term on the right. When the two derivatives in $\mathscr{L}_{z}$ are applied to the factor $r \ln r$, the following term arises:

$$
\frac{\delta^{3}}{r} \frac{f_{0}^{2}}{\Theta_{1}^{\prime}} M_{1}\left(\boldsymbol{e}_{r} \cdot \frac{\partial \boldsymbol{X}^{(1)}}{\partial z}\right)^{2} .
$$

The corresponding contribution to $\psi_{2}^{2 d}$ will be linear in $r$. All other contributions will be at least as smooth near $r=0$ as $r^{2} \ln r$. As a consequence, the source term in the equation for the remainder, i.e.

$$
\left(\nabla_{\|}^{2}+\mathscr{L}_{z}\right) \psi_{3}^{*}=-\mathscr{L}_{z} \psi_{2}^{2 d}-q_{r},
$$

is no longer singular at $r=0$ and $\psi_{3}^{*}$ will be smooth and thus lend itself to accurate numerical solution.

\section{Appendix C. Further details of the core solution}

\section{C.1. Homogeneous solutions to the second-order core streamfunction equation}

There are two linearly independent homogeneous solutions to (4.31). Because $\zeta_{\widehat{r}}^{(0)} \rightarrow 0$ as $\widehat{r} \rightarrow 0$ and $\widehat{r} \rightarrow \infty$, the operator on the left of (4.31) reduces to $\partial_{\widehat{r} r}+(1 / \widehat{r}) \partial_{\hat{r}}-1 / \widehat{r}^{2}$, with homogeneous solutions $\widehat{r}$ and $\widehat{r}^{-1}$, in these limits. One of the homogeneous solutions to (4.31) is of the form $C_{1 k} u_{\theta}^{(0)}$ with $C_{1 k}=$ const. It is proportional to $\widehat{r}$ near $\widehat{r}=0$ and to $\widehat{r}^{-1}$ as $\widehat{r} \rightarrow \infty$. The other homogeneous solution is singular at $\widehat{r}=0$ and must therefore be discarded.

\section{C.2. Higher harmonics of the leading-order core solutions}

Here we provide some considerations that were omitted from the main text so as to not distract from the principal line of thought.

Since $\widehat{p}^{(4)}$ is axisymmetric (see $(4.14 b)$ ), the vertical momentum balance in (4.17) implies that the cirumferential average is trivially hydrostatic, i.e.

$$
\widehat{\rho}_{0}^{(4)}=-\frac{\partial \widehat{p}^{(4)}}{\partial z},
$$

and that all circumferential harmonics higher than the first of $\widehat{\rho}^{(4)}$ and (via the equation of state (4.20)) of $\widehat{\Theta}^{(4)}$ vanish identically, i.e.

$$
\widehat{\Theta}_{l k}^{(4)}=-\frac{\widehat{\rho}_{l k}^{(4)}}{\rho_{0}}=0 \quad \text { for } l \geqslant 2 ; k=1,2 .
$$

Thus, $\widehat{\Theta}^{(4)}$ has no circumferential harmonics higher than the first. This allows us subsequently to obtain closed expressions for the harmonics of the second-order horizontal flow despite the presence of the nonlinear advection terms, which would otherwise couple all Fourier modes through triad interactions and render the equation system unclosed.

All higher harmonics $\widehat{w}_{l k}^{(1)}$ for $l \geqslant 2$ obey equations such as $(4.23 b)$ without the terms involving $\widehat{\Theta}_{l k^{*}}^{(4)}$ because these vanish identically (see (C2)). Thus, in an adiabatic vortex $\widehat{w}_{l k}^{(1)}=0$ for $l \geqslant 2$ and $k \in\{1,2\}$. Since we wish to allow only source terms $Q_{\Theta}$ that do not principally alter the solution structure relative to the adiabatic case, we assume

$$
Q_{\Theta, l k}^{(2)}=0 \quad \text { and hence } \quad \widehat{w}_{l k}^{(1)}=0 \quad \text { for }(l \geqslant 2 ; k=1,2) .
$$




\section{C.3. First-order horizontal momentum balance}

The radial momentum balance at $O\left(\delta^{-2}\right),(4.15 a)$, yields a higher-order gradient wind balance for $\widehat{p}_{0}^{(5)}$ after circumferential averaging,

$$
\frac{1}{\rho_{0}} \frac{\partial \widehat{p}_{0}^{(5)}}{\partial \widehat{r}}-\frac{2 u_{\theta}^{(0)} u_{\theta}^{(1)}}{\widehat{r}}-f_{0} u_{\theta}^{(1)}=0
$$

The first Fourier modes of (4.15) reveal the mode-one components of $\widehat{p}^{(5)}$ to be in geostrophic balance with the Coriolis force due to the leading-order vortex motion, i.e.

$$
\widehat{p}_{11}^{(5)}=-\widehat{r} \rho_{0} f_{0} \frac{\mathrm{d} X_{0}}{\mathrm{~d} t}, \quad \widehat{p}_{12}^{(5)}=\widehat{r} \rho_{0} f_{0} \frac{\mathrm{d} Y_{0}}{\mathrm{~d} t} .
$$

All higher harmonics of $\widehat{p}^{(5)}$ are found to vanish identically.

\section{C.4. Higher harmonics of the second-order horizontal flow}

Here we first define

$$
\mathscr{D}_{j}=\left(\frac{\partial^{2}}{\partial \widehat{r}^{2}}+\frac{1}{\widehat{r}} \frac{\partial}{\partial \widehat{r}}-\frac{j^{2}}{\widehat{r}^{2}}\right),
$$

and then, in analogy with the derivations in $\S 4.4$, arrive at

$$
\begin{gathered}
4 \mathscr{D}_{2}\left[\phi_{21}^{(2)}\right]=\frac{\partial X^{(1)}}{\partial z}\left[\frac{\partial}{\partial \widehat{r}}+\frac{1}{\widehat{r}}\right] \widehat{w}_{11}^{(1)}+\frac{\partial Y^{(1)}}{\partial z}\left[\frac{\partial}{\partial \widehat{r}}+\frac{1}{\widehat{r}}\right] \widehat{w}_{12}^{(1)}, \\
4 \mathscr{D}_{2}\left[\phi_{22}^{(2)}\right]=\frac{\partial X^{(1)}}{\partial z}\left[\frac{\partial}{\partial \widehat{r}}+\frac{1}{\widehat{r}}\right] \widehat{w}_{12}^{(1)}+\frac{\partial Y^{(1)}}{\partial z}\left[-\frac{\partial}{\partial \widehat{r}}+\frac{1}{\widehat{r}}\right] \widehat{w}_{11}^{(1)},
\end{gathered}
$$

and

$$
\mathscr{D}_{j}\left[\phi_{j k}^{(2)}\right]=0 \quad \text { for } j \geqslant 3 ; k=1,2 .
$$

The second harmonics, $\phi_{2 k}^{(2)}$, can be solved for analytically given the asymmetries of $\widehat{w}^{(1)}$ and the components of the tilt (Bronstein \& Semendjajew 1979). Just as for the first harmonics, these solutions should be determined under the constraint that the divergent part of the horizontal flow has to vanish at large $\widehat{r}$ consistent with the non-divergent outer flow. For higher harmonics mass conservation is guaranteed if $\phi_{j k}^{(2)} \equiv 0$ for $j \geqslant 3 ; k=1,2$.

In analysing the second and higher harmonics of the streamfunction we find

$$
\left(\frac{\partial^{2}}{\partial \widehat{r}^{2}}+\frac{1}{\widehat{r}} \frac{\partial}{\partial \widehat{r}}-\left[\frac{\zeta_{\widehat{r}}^{(0)}}{u_{\theta}^{(0)}}+\frac{4}{\widehat{r}^{2}}\right]\right) \psi_{22}^{(2)}=\frac{(-1)^{k^{*}}}{u_{\theta}^{(0)}}(\mathscr{H}+\mathscr{I}+\mathscr{J}+\mathscr{Q})_{2 k^{*}},
$$

where $k^{*}=3-k$, and

$$
\begin{gathered}
\mathscr{H}_{2 k}=\frac{1}{2} \frac{\partial}{\partial \widehat{r}}\left(\widehat{r}_{2 k}^{(1)} \frac{\partial u_{\theta}^{(0)}}{\partial z}\right), \\
\mathscr{I}_{2 k}=\frac{1}{2}\left[\widehat{r}\left(\zeta^{(0)}+f_{0}\right)\right] \mathscr{D}_{2} \phi_{2 k}^{(2)}, \\
\mathscr{J}_{2 k}=\frac{1}{2} \frac{\partial \phi_{2 k}^{(2)}}{\partial \widehat{r}}\left(r \frac{\partial \zeta^{(0)}}{\partial \widehat{r}}\right), \\
\mathscr{Q}_{2 k}=\left[-\frac{1}{4} \frac{\partial}{\partial \widehat{r}}\left(\widehat{r} \frac{\partial u_{\theta}^{(0)}}{\partial \widehat{r}}[\cdot]\right)+\frac{u_{\theta}^{(0)}}{2 \widehat{r}}\right] \mathscr{X}_{2 k},
\end{gathered}
$$


with

$$
\mathscr{X}_{21}=\left(\frac{\partial X^{(1)}}{\partial z} \widehat{w}_{11}^{(1)}+\frac{\partial Y^{(1)}}{\partial z} \widehat{w}_{12}^{(1)}\right), \quad \mathscr{X}_{22}=\left(\frac{\partial X^{(1)}}{\partial z} \widehat{w}_{12}^{(1)}-\frac{\partial Y^{(1)}}{\partial z} \widehat{w}_{11}^{(1)}\right) .
$$

The second harmonics of the horizontal velocity depend on the first Fourier modes and the vortex tilt, which do not depend on the second harmonics in return. Thus we will not need the second and higher harmonics to arrive at our main results, the evolution equations for the leading-order core structure and the vortex tilt. Yet we will need the large $-\widehat{r}$ behaviour of the second harmonics in constructing consistently matched asymptotic solutions for the vortex core and outer flow up to and including terms of $O(\delta)$.

In preparation for this step we consider the large- $\widehat{r}$ behaviour of homogeneous solutions to (C9). Since the derivative of the leading-order vorticity, $\zeta_{\vec{r}}^{(0)}$, in (C9) vanishes for large $\widehat{r}$ by assumption, these homogeneous solutions satisfy

$$
\left(\frac{\partial^{2}}{\partial \widehat{r}^{2}}+\frac{1}{\widehat{r}} \frac{\partial}{\partial \widehat{r}}-\frac{4}{\widehat{r}^{2}}\right) \psi_{2 k, h}^{(2)}=o(1) \quad \text { as } \widehat{r} \rightarrow \infty,
$$

and one finds that

$$
\psi_{2 k, h}^{(2)}=\psi_{2 k}^{*} \widehat{r}^{2}(1+o(1)) \quad \text { as } \widehat{r} \rightarrow \infty
$$

with $\psi_{2 k}^{*}=$ const is consistent with this behaviour. The amplitudes $\psi_{2 k}^{*}$ of the corresponding homogeneous contributions to $\psi_{2 k}^{(2)}$ are determined in the course of matching the inner and outer solutions in $\S 5$.

The higher harmonics of the streamfunction satisfy

$$
\left(\frac{\partial^{2}}{\partial \widehat{r}^{2}}+\frac{1}{\widehat{r}} \frac{\partial}{\partial \widehat{r}}-\left[\frac{\zeta_{\widehat{r}}^{(0)}}{u_{\theta}^{(0)}}+\frac{j^{2}}{\widehat{r}^{2}}\right]\right) \psi_{j k}^{(2)}=0 \quad \text { for } j \geqslant 3 ; k=1,2
$$

and these equations are trivially solved by $\psi_{j k}^{(2)} \equiv 0$.

\section{Appendix D. Linearization of the outer flow near the centreline}

As the outer flow regular velocity is divergence-free its local linearization can be expressed in terms of a local quadratic streamfunction,

$$
\boldsymbol{r e}_{r} \cdot\left(\nabla_{\|} \boldsymbol{u}^{*}\right)_{0}=-\boldsymbol{k} \times \nabla_{\|}\left(-\frac{r^{2} \zeta_{0}^{*}}{2}+\psi_{2}^{*}\right)
$$

where

$$
\psi_{2}^{*}(r, \theta)=r^{2}\left(\psi_{21}^{*} \sin (2 \theta)+\psi_{22}^{*} \cos (2 \theta)\right) .
$$

Here $\zeta_{0}^{*}$ is the vorticity of the regular part of the flow at $\boldsymbol{X}_{0}$, and $\psi_{21}^{*}, \psi_{22}^{*}$ define the components of the symmetric part of the velocity gradient, i.e. of the local irrotational strain tensor. A straightforward calculation shows that

$$
\left(\left(\nabla_{\|} \boldsymbol{u}^{*}\right)_{0}\right)=-\left(\begin{array}{cc}
-\psi_{21}^{*} & \psi_{22}^{*}+\zeta_{0}^{*} \\
\psi_{22}^{*}-\zeta_{0}^{*} & \psi_{21}^{*}
\end{array}\right)
$$

The representation in (D 1), (D 2) implies (5.7). 
Appendix E. Large- $\widehat{r}$ behaviour of the operator in (5.10), (5.11)

Consider the solution operator for the first-Fourier-mode streamfunction of the inner solution in (4.35), i.e.

$$
\mathscr{L}_{u}[\mathscr{F}]=\frac{u}{\widehat{r}} \int_{0}^{\widehat{r}} \frac{1}{\bar{r} u^{2}} \int_{0}^{\bar{r}} r^{\prime} \mathscr{F}\left(r^{\prime}\right) \mathrm{d} r^{\prime} \mathrm{d} \bar{r} .
$$

Assume that $u=(\Gamma / 2 \pi \widehat{r})(1+o(1))$ and $\widehat{r}^{2} \mathrm{~d} u / \mathrm{d} \widehat{r}=-\left(\Gamma / 2 \pi \widehat{r}^{2}\right)(1+o(1))$ as $\widehat{r} \rightarrow \infty$. Following de L'Hôpital's rule we have

$$
\lim _{\widehat{r} \rightarrow \infty} \mathscr{L}_{u}[f](\widehat{r})=\lim _{\widehat{r} \rightarrow \infty} \frac{\int_{0}^{\widehat{r}} r^{\prime} f\left(r^{\prime}\right) \mathrm{d} r^{\prime}}{\widehat{r} u^{2}\left(\frac{1}{u}-\frac{\widehat{r} u^{\prime}}{u^{2}}\right)}=\frac{\pi}{\Gamma} \int_{0}^{\infty} r f(r) \mathrm{d} r
$$

if the limit on the right exists. For the four source terms of the equations for the $\psi_{1 k}^{(1)}$ in (4.35) we now need to investigate the convergence of this last integral.

For $\mathscr{H}_{1 k}$, defined in (4.33), we recall the solution for $\widehat{w}_{1 k}^{(1)}$ in (4.25). Since we consider the diabatic source term $Q_{\Theta}^{(2)}$ as given externally here, we may assume it to decay sufficiently rapidly so that whenever it occurs in the integrand in $\mathscr{L}_{u}[f]$, the inner integral will converge. Then, the term in $\widehat{w}_{1 k}^{(1)}$ with the slowest decay is proportional to $u_{\theta}^{(0)^{2}} \widehat{r} \sim \widehat{r}^{-3}$, since $u_{\theta}^{(0)} \sim \widehat{r}^{-1}$ for large $\widehat{r}$. This implies $\widehat{r} \mathscr{H}_{1 k} \sim \widehat{r}^{-3}$, and last integral in (E 2) will converge for $f=\mathscr{H}_{1 k}$. Analogously, $\widehat{r} \mathscr{Q}_{1 k} \sim \widehat{r}^{-4}$ and the integral converges.

For the convergence of the integral with $f=\mathscr{J}_{1 k}$ we have to assume in addition that $\partial^{2} u_{\theta}^{(0)} / \partial \widehat{r}^{2}=\Gamma /\left(\pi \widehat{r}^{3}\right)(1+o(1))$. In that case, since $\phi_{1 k}^{(2)} \rightarrow 0$ under the conditions formulated below (4.30) (checked via two-fold application of de L'Hôpital's rule) we also have $\partial \phi_{1 k}^{(2)} / \partial \widehat{r} \rightarrow 0$ and $\widehat{r} \mathscr{J}_{1 k}=O\left(\widehat{r}^{-2}\right)$ as $\widehat{r} \rightarrow \infty$, and the integral converges.

Finally with $\widehat{w}_{1 k}^{(1)} \sim \widehat{r}^{-3}$, the term with slowest decay in $\mathscr{I}_{1 k}$ from (4.33) is the one involving the factor $\widehat{r} f_{0}$, and we have $\widehat{r} \mathscr{I}_{1 k} \sim \widehat{r}^{-1}$ for large $\widehat{r}$ and the last integral in (E 2) diverges. More precisely,

$$
\widehat{r} \mathscr{I}_{1 k}=-\frac{I_{1 k}}{\widehat{r}}(1+o(1)) \quad \text { as } \widehat{r} \rightarrow \infty,
$$

where

$$
I_{1 k}=\frac{f_{0}^{2}}{8 \pi^{2} \rho_{0}} \frac{\partial}{\partial z}\left(\frac{\rho_{0} \Gamma^{2}}{\Theta_{1}^{\prime}}(-1)^{k} \frac{\partial X_{k}^{(1)}}{\partial z}\right) .
$$

By adding and subtracting this dominant behaviour we find a decomposition of $\mathscr{L}_{u}\left[\mathscr{I}_{1 k}\right](\widehat{r})$ into a logarithmically diverging term plus a finite contribution, namely

$$
\mathscr{L}_{u}\left[\mathscr{I}_{1 k}\right](\widehat{r})=-\frac{\pi I_{1 k}}{\Gamma} \ln \widehat{r}+L\left[\widetilde{\mathscr{I}}_{1 k}\right]+o(1) \quad(\widehat{r} \rightarrow \infty)
$$

where

$$
\widetilde{\mathscr{I}_{1 k}}=\mathscr{I}_{1 k}+H(\widehat{r}-1) I_{1 k} / \widehat{r}^{2}
$$

is the regularized version of $\mathscr{I}_{1 k}$ with $H(\cdot)$ the Heaviside step function. 


\section{Appendix F. Centreline motion for the barotropic, adiabatic case}

We start from the first-order centreline equation of motion in $(5.17 b)$, namely

$$
\frac{\partial \boldsymbol{X}^{(1)}}{\partial t}(t, z)=\boldsymbol{u}_{0}^{(1 / 2)}+\boldsymbol{X}^{(1)} \cdot\left(\nabla_{\|} \boldsymbol{u}\right)_{0}^{(0)}-\ln \frac{1}{\delta}\left(\boldsymbol{k} \times \boldsymbol{M}_{1}\right)+(\boldsymbol{k} \times \boldsymbol{\Psi})
$$

where

$$
\begin{gathered}
\boldsymbol{M}_{1}=\frac{f_{0}^{2}}{4 \pi \rho_{0} \Gamma} \frac{\partial}{\partial z}\left(\frac{\rho_{0} \Gamma^{2}}{\Theta_{1}^{\prime}} \frac{\partial \boldsymbol{X}^{(1)}}{\partial z}\right) \\
\boldsymbol{\Psi}=\left(-\boldsymbol{i} L\left[\mathscr{H}_{11}+\widetilde{\mathscr{I}}_{11}+\mathscr{J}_{11}\right]+\boldsymbol{j} L\left[\mathscr{H}_{12}+\widetilde{\mathscr{I}}_{12}+\mathscr{J}_{12}\right]\right)
\end{gathered}
$$

furthermore,

$$
L\left[\mathscr{H}_{1 k}+\widetilde{\mathscr{I}_{1 k}}+\mathscr{J}_{1 k}\right]=R_{1}\left[W T_{k}\right]+R_{2}\left[\frac{1}{\rho_{0}} \frac{\partial}{\partial z}\left(\rho_{0} W T_{k}\right), \frac{1}{\rho_{0}} \frac{\partial}{\partial z}\left(\rho_{0} \widehat{W} T_{k}\right)\right] .
$$

Here we have used (4.25) for the diabatic case, so that

$$
\widehat{w}_{1 k}^{(1)}=W T_{k} \quad \text { with } W=\frac{1}{\Theta_{1}^{\prime}} \frac{u_{\theta}^{(0)^{2}}}{\widehat{r}}\left(\frac{u_{\theta}^{(0)}}{\widehat{r}}+f_{0}\right)
$$

and where we have defined the abbreviations

$$
\widehat{W}=\frac{\Gamma^{2}}{4 \pi^{2}} \frac{f_{0}^{2}}{\Theta_{1}^{\prime}}, \quad T_{1}=-\frac{\partial X_{C}^{(1)}}{\partial z}, \quad T_{2}=+\frac{\partial Y_{C}^{(1)}}{\partial z},
$$

and

$$
\begin{aligned}
R_{1}[W]= & \frac{\pi}{\Gamma} \int_{0}^{\infty} r \frac{\partial}{\partial r}\left(r W \frac{\partial u_{\theta}^{(0)}}{\partial z}\right) \mathrm{d} r \\
R_{2}[W, \widehat{W}]= & \frac{\pi}{\Gamma} \int_{0}^{\infty}\left(-r^{2}\left[\zeta^{(0)}+f_{0}\right] W+\frac{H(r-1)}{r} \widehat{W}\right) \mathrm{d} r \\
& -\frac{\pi}{\Gamma} \int_{0}^{\infty}\left(r^{2} \frac{\partial \zeta^{(0)}}{\partial r}\right) \frac{\partial}{\partial r}\left(r \int_{0}^{r} \frac{1}{\bar{r}^{3}}\left[\int_{0}^{\bar{r}} \overline{\bar{r}}^{2} W \mathrm{~d} \overline{\bar{r}}\right] \mathrm{d} \bar{r}\right) \mathrm{d} r \\
& +\frac{\pi}{\Gamma} \int_{0}^{\infty}\left(r^{2} \frac{\partial \zeta^{(0)}}{\partial r}\right) \mathrm{d} r \int_{0}^{\infty} \frac{1}{\bar{r}^{3}}\left[\int_{0}^{\bar{r}} \overline{\bar{r}}^{2} W \mathrm{~d} \overline{\bar{r}}\right] \mathrm{d} \bar{r} .
\end{aligned}
$$

Finally, we find

$$
\boldsymbol{\Psi}=\tilde{A} \frac{\partial^{2} \boldsymbol{X}^{(1)}}{\partial z^{2}}+\tilde{B} \frac{\partial \boldsymbol{X}^{(1)}}{\partial z}
$$

where

$$
\tilde{A}=R_{2}[W, \widehat{W}] \quad \text { and } \quad \tilde{B}=R_{1}[W]+\frac{1}{\rho_{0}} \frac{\partial}{\partial z}\left(\rho_{0} R_{2}[W, \widehat{W}]\right) .
$$

Collecting all results from this section we obtain the equation of motion for $\boldsymbol{X}^{(1)}$ as announced in (6.2), (6.3). 


\section{REFERENCES}

Afanasyev, YA. D. \& Peltier, W. R. 1998 Three-dimensional instability of anticyclonic swirling flow in rotating fluid: laboratory experiments and related theoretical predictions. Phys. Fluids 10 (12), 3194-3202.

Bronstein, I. \& Semendjajew, K. 1979 Taschenbuch der Mathematik. Harri Deutsch.

CAllegari, A. J. \& Ting, L. 1978 Motion of a curved vortex filament with decaying vortical core and axial velocity. SIAM J. Appl. Maths 35 (1), 148-175.

Charney, J. G. \& Eliassen, A. 1964 On the growth of the hurricane depression. J. Atmos. Sci. 21, 68-75.

ECKhaus, W. 1979 Asymptotic Analysis of Singular Perturbations, vol. 9. North-Holland.

EgGER, J. 1992 Point vortices in a low-order model of barotropic flow on the sphere. $Q . J . R$. Meteorol. Soc. 118, 533-552.

ELIASSEN, A. 1952 Slow thermally or frictionally controlled meridional circulation in a circular vortex. Astrophys. Norv. 5, 19-60.

Emanuel, K. A. 1991 The theory of hurricanes. Annu. Rev. Fluid Mech. 23, 179-196.

Emanuel, K. A. 2003 Tropical cyclones. Annu. Rev. Earth Planet. Sci. 31, 75-104.

FRANK, W. M. \& Ritchie, E. A. 1999 Effects of environmental flow upon tropical cyclone structure. Mon. Weath. Rev. 127, 2044-2061.

JONES, S. 1995 The evolution of vortices in vertical shear i: initially barotropic vortices. $Q . J . R$. Meteorol. Soc. 121, 821-851.

JONES, S. 2000 The evolution of vortices in vertical shear ii: large-scale asymmetries. $Q$. J. $R$. Meteorol. Soc. 126, 3137-3159.

JONES, S. 2004 On the ability of dry tropical-cyclone-like vortices to withstand vertical shear. J. Atmos. Sci 61, 114-119.

KELlER, J. B. \& WARD, W. 1996 Asymptotics beyond all orders for a low Reynolds number flow. J. Engng Maths 30, 253-265.

KLEIN, R. 2010 Scale-dependent asymptotic models for atmospheric flows. Annu. Rev. Fluid Mech. 42, 249-274.

KLEIN, R. \& MAJDA, A. J. 1991 Self-stretching of a perturbed vortex filament i: the asymptotic equation for deviations from a straight line. Physica D 49, 323-352.

LING, G. \& TING, L. 1988 Two-time scales inner solutions and motion of a geostrophic vortex. Sci. Sin. XXXI (7).

McWilliams, J. C., Graves, L. P. \& Montgomery, M. T. 2003 A formal theory for vortex Rossby waves and vortex evolution. Geophys. Astrophys. Fluid Dyn. 97 (4), 275-309.

MiKUSKY, E. 2007 On the structure of concentrated atmospheric vortices in a gradient wind regime and its motion on synoptic scales. PhD thesis, Universität Hamburg, Fachbereich Geowissenschaften.

Montgomery, M. T. \& Kallenbach, R. K. 1997 A theory for vortex Rossby waves and its application to spiral bands and intensity changes in hurricanes. Q. J. R. Meteorol. Soc. 123, 435-465.

MoriKaWA, G. K. 1960 Geostrophic vortex motion. J. Meteorol. 17, 148-158.

Pedlosky, J. 1987 Geophysical Fluid Dynamics, 2nd edn. Springer.

Plougonven, R. \& Zeitlin, V. 2002 Internal gravity wave emission from a pancake vortex: An example of wave-vortex interaction in strongly stratified flows. Phys. Fluids 14, 1259-1268.

Reasor, P. D. \& Montgomery, M. T. 2001 Three-dimensional alignment and corotation of weak, tc-like vortices via linear vortex rossby waves. J. Atmos. Sci 58, 2306-2330.

Reasor, P. D., Montgomery, M. T. \& Grasso, L. D. 2004 A new look an the problem of tropical cyclones in vertical shear flow. J. Atmos. Sci. 61 (1), 3-22.

REZnIK, G. M. 1992 Dynamics of singular vortices on a beta-plane. J. Fluid Mech. 240, 405-432.

REZNIK, G. M. \& GRIMSHAW, R. 2001 Ageostrophic dynamics of an intense localized vortex on a $\beta$-plane. J. Fluid Mech. 443, 351-376.

REZNIK, G. \& KIZNER, Z. 2007a Two-layer quasi-geostrophic singular vortices embedded in a regular flow. Part 1. Invariants of motion and stability of vortex pairs. J. Fluid Mech. 584, 185-202. 
REZNIK, G. \& KIZNER, Z. $2007 b$ Two-layer quasi-geostrophic singular vortices embedded in a regular flow. Part 2. Steady and unsteady drift of individual vortices on a beta-plane. J. Fluid Mech. 584, 203-223.

RICCA, R. L. 1991 Rediscovery of da rios equations. Nature 352, 561-562.

Schecter, D. A. \& Montgomery, M. T. 2003 On the symmetrization rate of an intense geophysical vortex. Dyn. Atmos. Oceans 37, 55-88.

Schecter, D. A. \& Montgomery, M. T. 2004 Damping and pumping of a vortex Rossby wave in a monotonic cyclone: critical layer stirring versus inertia-buoyancy wave emission. Phys. Fluids 16, 1334-1348.

Schecter, D. A. \& Montgomery, M. T. 2006 Conditions that inhibit the spontaneous radiation of spiral inertia-gravity waves from an intense mesoscale cyclone. J. Atmos. Sci 63, 435-456.

Schecter, D. A. \& Montgomery, M. T. 2007 Waves in a cloudy vortex. J. Atmos. Sci 64, 314-337.

Schecter, D. A., Montgomery, M. T. \& Reasor, P. D. 2002 A theory for the vertical alignment of a quasigeostrophic vortex. J. Atmos. Sci 59, 150-168.

Schubert, W. H. \& HACK, J. J. 1983 Transformed Eliassen balanced vortex model. J. Atmos. Sci 39, $1687-1697$.

Shapiro, L. J. \& MONTGOMERY, M. T. 1993 A three-dimensional theory for rapidly rotating vortices. J. Atmos. Sci 50, 3322-3335.

SMith, R. K. 1991 An analytic theory of tropical-cyclone motion in a barotropic shear flow. Q. J. R. Meteorol. Soc. 47, 685-714.

Smith, R. \& Montgomery, M. T. 2010 Hurricane boundary-layer theory. Q. J. R. Meteorol. Soc. 136, 1665-1670.

SMith, R. K. \& UlRich, W. 1990 An analytic theory of tropical cyclone motion using a barotropic model. J. Atmos. Sci 47, 1973-1986.

STEWART, H. J. 1943 Periodic properties of the semi-permanent atmospheric pressure systems. Q. Appl. Maths 1, 262-267.

Ting, L., Klein, R. \& KNio, O. M. 2007 Vortex Dominated Flows: Analysis and Computation for Multiple Scales, Series in Applied Mathematical Sciences, vol. 116. Springer.

TING, L. \& LING, G. 1983 Studies on the motion and core structure of a geostrophic vortex. In Proc. 2nd Asian Congress of Fluid Mechanics, pp. 900-905. Science Press.

VAN DYKe, M. 1964 Perturbation Methods in Fluid Mechanics. Academic.

Wirth, V. \& DUnkerton, T. J. 2009 The dynamics of eye formation and maintenance in axisymmetric diabatic vortices. J. Atmos. Sci 66, 3601-3620. 